\title{
Analyzing scenery images by monotonic tree
}

\author{
Yuqing Song and Aidong Zhang
}

Department of Computer and Information Science, The University of Michigan at Dearborn, 4901 Evergreen, Dearborn, Michigan 48128, USA

\begin{abstract}
Content-based image retrieval (CBIR) has been an active research area in the last ten years, and a variety of techniques have been developed. However, retrieving images on the basis of low-level features has proven unsatisfactory, and new techniques are needed to support high-level queries. Research efforts are needed to bridge the gap between high-level semantics and low-level features. In this paper, we present a novel approach to support semantics-based image retrieval. Our approach is based on the monotonic tree, a derivation of the contour tree for use with discrete data. The structural elements of an image are modeled as branches (or subtrees) of the monotonic tree. These structural elements are classified and clustered on the basis of such properties as color, spatial location, harshness and shape. Each cluster corresponds to some semantic feature. This scheme is applied to the analysis and retrieval of scenery images. Comparisons of experimental results of this approach with conventional techniques using low-level features demonstrate the effectiveness of our approach.
\end{abstract}

Keywords: Content-based image retrieval - Image feature extraction - Annotation - Semantics retrieval - Monotonic tree

\section{Introduction}

With the enormous growth of image databases, there is an urgent need to build efficient and effective image retrieval systems. Content-based image retrieval (CBIR) offers a promising technology to address this need, and a variety of CBIR techniques have been developed. In particular, content-based image retrieval using low-level features such as color $[42,37$, $26]$, texture $[21,36,35,41,20]$, shape $[43,22,12,23,24,13,9$, $15,47,48,34,44,33]$ and others $[28,37,2,14,7]$ has been well studied. Various image-querying systems, including QBIC [10], VisualSeek [37], PhotoBook [27], Netra [18], and Virage [5], have been built, using low-level features for general or specific image-retrieval tasks.

However, retrieving images via low-level features has proven to be unsatisfactory. Effective and precise image retrieval by semantics still remains an open problem because of the extreme difficulty of fully characterizing images. Theories and approaches for querying and browsing images based on image semantics become increasingly critical as web-based information retrieval grows in popularity. In recognizing the existing problems in the CBIR field, we believe that research efforts are needed to bridge the gap between the high-level semantics of interest to users and the low-level features which can be efficiently extracted.

J. P. Eakins [8] classified image features into three levels, ranging from the highly concrete to the very abstract. For our purpose, we classify image features in three levels, as follows:

- Primitive level: primitive features include color, texture, shape, and the spatial location of image elements.

- Local semantic level: local semantic features describe the presence of individual objects in images. Two examples of queries by local semantic features are "find pictures with a bridge" (object of a given type) and "find pictures with sky and trees" (combination of objects).

- Thematic level (or global semantic level): thematic features describe the global meanings or topics of images. Two examples of queries at this level are "find pictures of a Chinese garden" and "find pictures of an earthquake." The thematic features of an image are based on all objects in the image along with their spatial relationships. Highlevel reasoning is needed to derive the global meaning of all objects in the scene, and to determine the topic of the image.

Most existing CBIR systems are based on primitive features [10,27,37,18]. Although some approaches [11,45,4] have been proposed for image retrieval by semantic features, there is currently no CBIR system which works effectively with semantic features. Due to the difficulty of detection and recognition of general objects, there is a long way to go before we can have a CBIR system which can extract general semantic features from images. However, if we restrain ourselves to specific domains, we can achieve quick success.

In this paper, we focus on scenery images, a popular testbed for semantics extraction. For several reasons, scenery images are easier to analyze than general images. First, scenery images contain a limited range of object types. Common scenery object types include sky, tree, building, mountain, lawn, water, and snow. Secondly, shape features, which are 

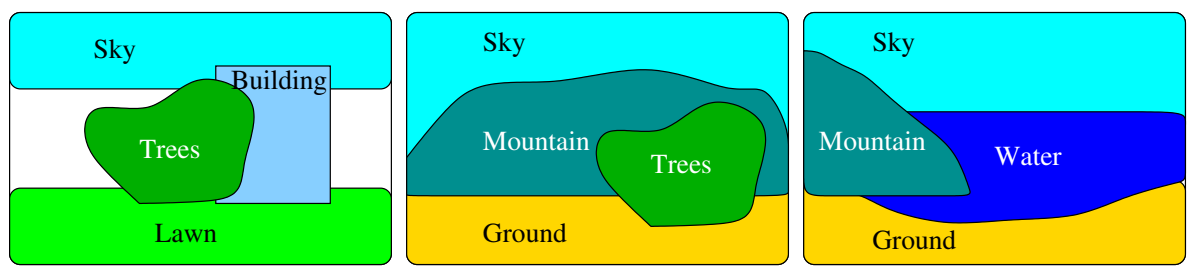

Fig. 1. Some styles of scenery images

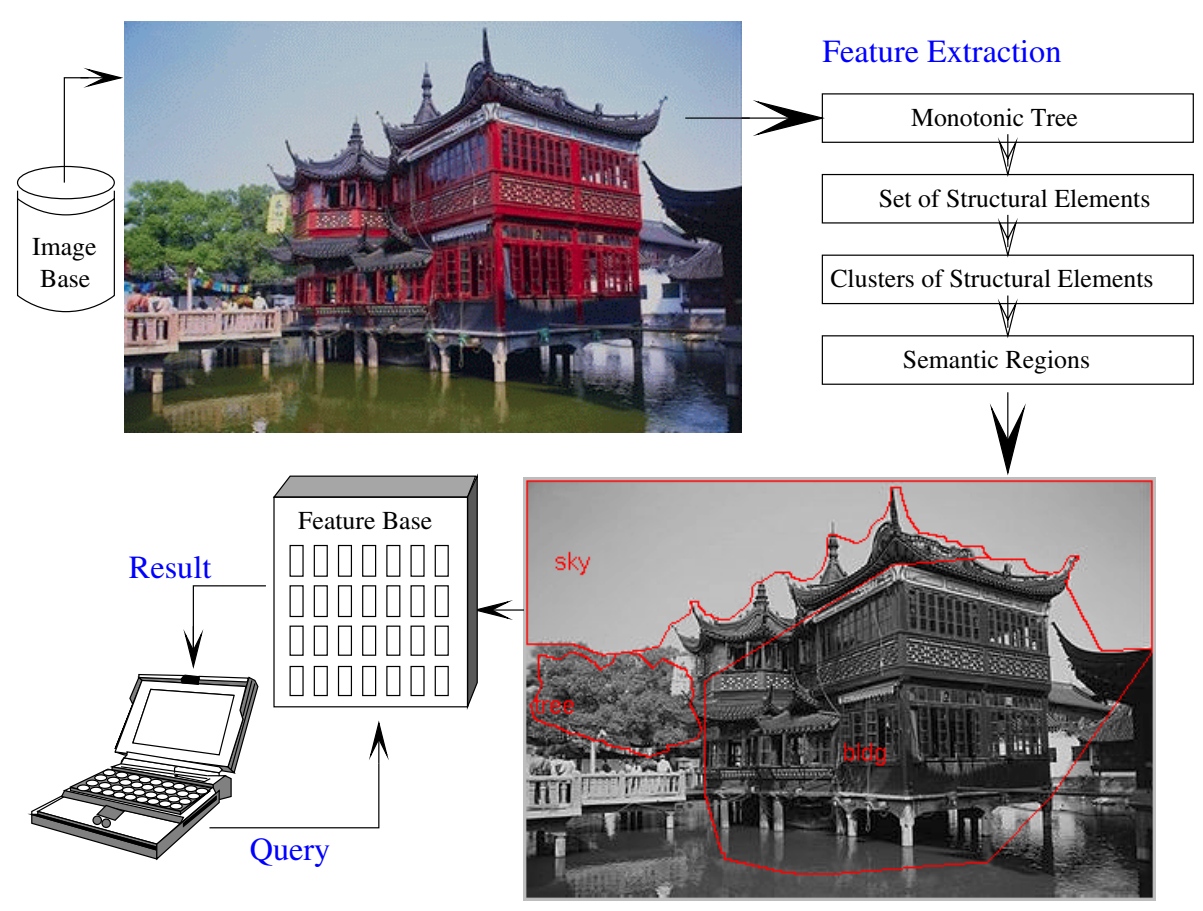

Fig. 2. SceneryAnalyzer architecture difficult to characterize and match, are less important than other low-level features in analyzing scenery images. Finally, scenery images often fall into a small number of typical patterns, such as those shown in Fig. 1. These typical patterns greatly simplify scene interpretation for scenery images.

In outdoor pictures, scenery features such as sky, trees, ground, and water are common types of background objects. Locating and interpreting background objects is essential to object recognition and image understanding. Among all objects in a natural image, it is quite common that a foreground object (or a group of foreground objects) indicates the theme of the image, while the background objects provide contextual and complementary information. Needless to say, successfully recognizing background objects in an image is a necessary step in determining the theme of the image. In addition, the contextual information provided by background objects helps, and in many cases is necessary, in recognizing foreground objects in two aspects:

- it can narrow down the possible types of foreground objects and make foreground object recognition more efficient; and

- it can be used to resolve the ambiguity in recognizing foreground objects.

Thus, finding effective ways to recognize common background objects will be a milestone in solving the problem of image understanding.

In this paper, we present a novel approach to support semantics-based image retrieval. This approach is based on the concept of the monotonic tree [39], a derivation of the contour tree for use with discrete data. The structural elements of an image are modeled as branches (or subtrees) of the monotonic tree of the image. These structural elements are classified and clustered on the basis of such properties as color, spatial location, harshness, and shape. Each cluster corresponds to some semantic feature. Following these steps, images can be automatically annotated with category keywords, including sky, building, tree, wave, lawn, water, snow, and ground, thus facilitating high-level (semantics-based) image querying and browsing.

A scenery-querying system, termed SceneryAnalyzer, has been built to demonstrate the effectiveness of this approach. SceneryAnalyzer handles six types of scenery features: sky, building, tree, water wave, placid water, and ground. The visual properties of water wave and placid water are structurally different, and they are therefore treated as separate types. The ground feature in images can be further split into snow, lawn, and other subtypes.

The process of generating the semantic keywords which support high-level scenery querying is shown in Fig. 2. Feature extraction is the central and unique component of this system. The extraction of features is accomplished off-line; each image is processed to extract semantic features, which are then stored in a feature vector. These semantic features are automatically annotated onto a gray copy of the original image. All feature vectors are stored in a feature base. 


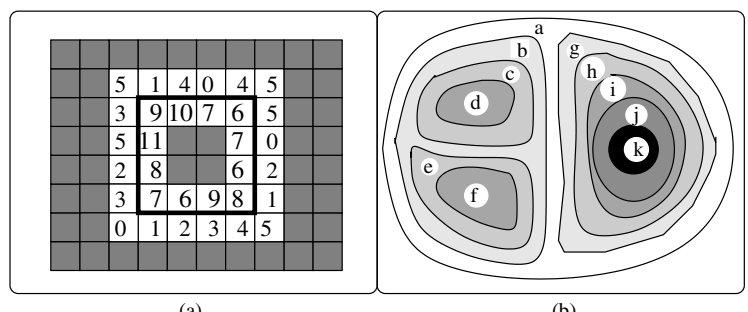

(a)

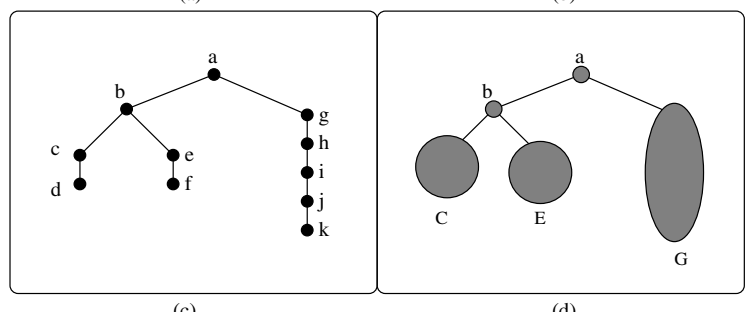

Fig. 3. a An outward-falling monotonic line (solid line), b a set of monotonic lines, $\mathbf{c}$ the monotonic tree, and $\mathbf{d}$ the topological monotonic tree

The body of this paper will be devoted to a presentation of the details of the approach to semantic feature extraction, including experimental results. Sect. 2 introduces the concept of the monotonic tree. In Sect. 3, techniques for extracting semantic features are described. Section 4 presents case studies of scenery features, while Sect. 5 offers a performance evaluation of the proposed approach. A summary and concluding remarks appear in Sect. 6.

\section{Monotonic tree}

\subsection{Introduction to the monotonic tree}

Contour trees $[25,32,46]$ have been used in geographic information systems (GIS) and medical imaging to display scalar data. For example, elevation in the landscape can be modeled by scalar data over the plane, and a contour (also called an isoline) is a line along which the elevation function assumes a constant value. Contours are defined only for continuous functions. For an image represented by discrete data, a continuous function is first defined through interpolation of the data. The contour tree is then defined on this continuous function.

In earlier work [39] we introduced a new concept, the monotonic line, which is defined directly on discrete data. An outward-falling/climbing monotonic line for a gray image is a boundary within the image which is characterized by higher/lower pixel values just inside the boundary than just outside. All monotonic lines in an image form a rooted tree, called a monotonic tree. A maximal sequence of uniquelyenclosing monotonic lines is called a monotonic slope. All monotonic slopes in an image form a topological monotonic tree. A monotonic slope is called outward-falling/climbing if all its monotonic lines are outward-falling/climbing. Figure 3 illustrates these concepts. For a color image, the monotonic tree and topological monotonic tree are constructed on its gray copy.

\subsection{Reduced monotonic tree}

The process presented in this paper employs a reduced monotonic tree, rather than the topological monotonic tree, because the reduced monotonic tree can be constructed recursively. In the monotonic tree of a gray image, the leaf nodes correspond to the boundaries of the local maximum/minimum regions in the image. Starting from the leaf nodes in the monotonic tree, we find the monotonic slopes which contain these leaf nodes. These monotonic slopes are the leaf nodes in the topological monotonic tree, which are called the slopes at level one. By removing these monotonic slopes, we produce a smoothed gray image. We then apply the same process to the smoothed image to generate the slopes at level two. The process is repeated recursively to produce a hierarchy of slopes. Together, these slopes form a reduced monotonic tree. ${ }^{1}$

Figures 4-6 illustrate an algorithm for the generation of a reduced monotonic tree. The algorithm consists of the following steps:

(1) Transfer the image to the hexagonal grid ${ }^{2}$ (as shown in Fig. 4). Denote the image on the hexagonal grid as $I=$ $(f, \Omega)$, where $\Omega$ is the domain of the image and $f$ is a function from $\Omega$ to $\mathbb{R}$. Let $f_{E}$ be the extended function of $f$ to the whole plane, which is equal to $f$ on $\Omega$, and assumes $-\infty$ out of $\Omega$.

(2) Find simply-connected regions ${ }^{3}$ where $f_{E}$ assumes local minimum or maximum values, as shown in Fig. 5a.

(3) For each local minimum/maximum, simply-connected region $X$, find the slope including $\partial X^{4}$, where a slope is a sequence of monotonic lines with each line uniquely and directly enclosing the next, as shown in Fig. 5b. The procedure for finding the slope including $\partial X$ is as follows:

(a) let $Y=X$ and $S=\{\partial Y\}$;

(b) if $Y=\Omega$, output $S$ and stop;

(c) if $\partial Y$ is outward-falling/climbing, let $v$ be the highest/lowest value assumed by pixels ${ }^{5}$ adjacent to $Y$;

(d) let $\left\{Z_{i}\right\}_{i=1}^{n}$ be the set of maximal connected regions such that (i) each $Z_{i}$ is adjacent to $Y$, and (ii) $f_{E}$ assumes the constant value $v$ over each $Z_{i}$. Let $Z=$ $\bigcup_{i=1}^{n} Z_{i}$

(e) if $\partial(Y \bigcup Z)$ is not a monotonic line, output $S$ and stop;

(f) let $Y=Y \bigcup Z$ and $S=S \bigcup\{\partial Y\}$; go to step (b).

All these slopes are nodes of the reduced monotonic tree, as shown in Fig. 6a.

(4) If only one slope is found in step (3) and it covers the whole image, stop. Otherwise, smooth the image by removing all the slopes found in step (3). Let $\left\{X_{i}\right\}_{i=1}^{n}$ be the set of regions covered by those slopes, respectively. For each

${ }^{1}$ The parent-child relation in the reduced monotonic tree is defined in a similar manner to that for the topological monotonic tree.

${ }^{2}$ Our theoretical definition of a monotonic tree is based on the hexagonal grid, which avoids the contiguity paradoxes of the square grid. A detailed discussion of the advantages of the hexagonal grid over the square one can be found in Snyder et al. [38].

${ }^{3}$ A simply-connected region is a connected region whose boundary is also connected. A finite connected region is simply-connected iff it has no hole.

${ }^{4} \partial X$ is the boundary of $X$.

${ }^{5}$ Here, by "a pixel assumes value $v$ ", we mean that $f_{E}$ assumes $v$ over this pixel. 


\begin{tabular}{|l|l|l|l|l|l|l|l|l|l|l|l|l|}
\hline 2 & 2 & 2 & 2 & 5 & 5 & 5 & 5 & 2 & 2 & 2 & 2 & 2 \\
\hline 2 & 2 & 2 & 5 & 7 & 7 & 7 & 5 & 5 & 2 & 2 & 2 & 2 \\
\hline 2 & 2 & 5 & 7 & 8 & 8 & 7 & 5 & 4 & 5 & 5 & 5 & 2 \\
\hline 2 & 5 & 7 & 8 & 9 & 8 & 7 & 4 & 3 & 3 & 3 & 5 & 2 \\
\hline 2 & 5 & 7 & 8 & 8 & 7 & 5 & 3 & 2 & 2 & 3 & 5 & 2 \\
\hline 2 & 5 & 7 & 7 & 7 & 5 & 3 & 2 & 2 & 3 & 4 & 5 & 2 \\
\hline 2 & 5 & 5 & 5 & 5 & 4 & 3 & 3 & 3 & 5 & 5 & 2 & 2 \\
\hline 4 & 7 & 2 & 4 & 5 & 5 & 5 & 5 & 5 & 4 & 2 & 2 & 2 \\
\hline 5 & 6 & 2 & 3 & 2 & 2 & 2 & 2 & 2 & 2 & 2 & 2 & 2 \\
\hline
\end{tabular}

(a)

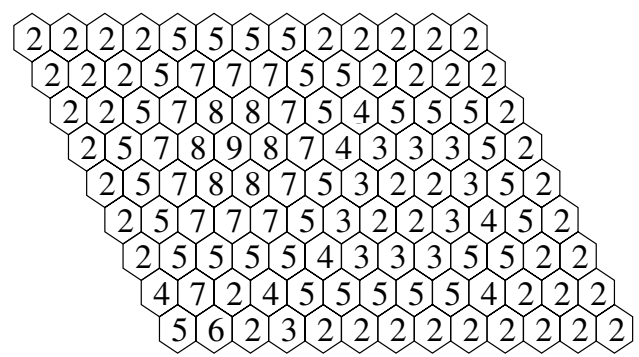

(b)

Fig. 4. a A gray image with a square grid (each integer represents a pixel value in the image), $\mathbf{b}$ the gray image transferred to a hexagonal grid

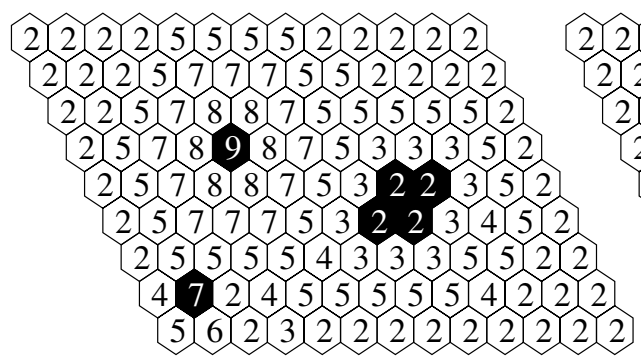

(a)

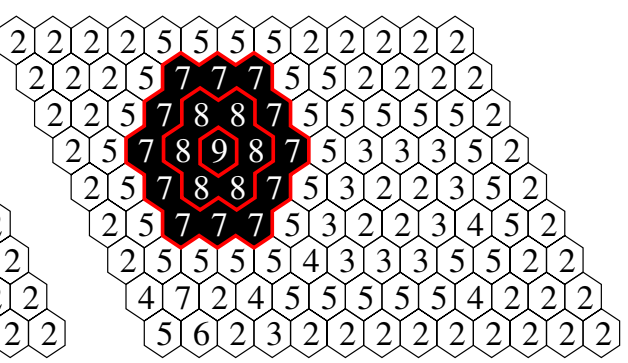

(b)

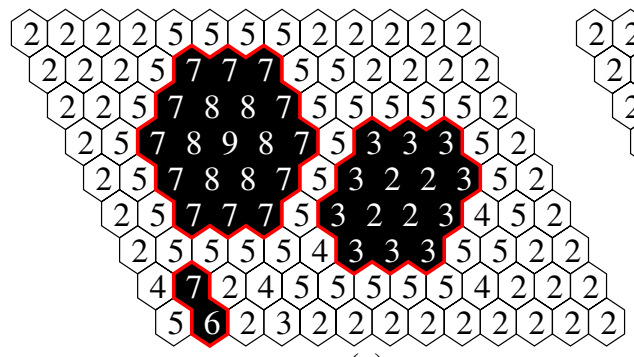

(a)

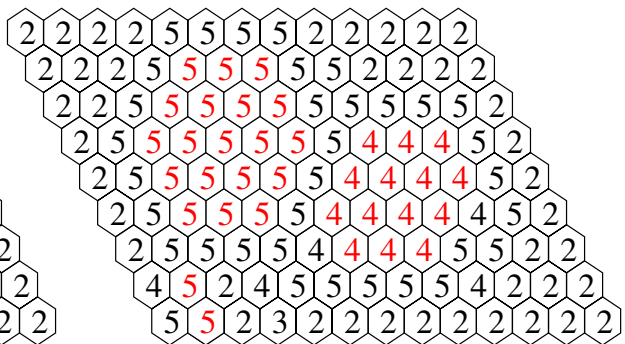

(b)
Fig. 5. a Simply connected regions where function $f_{E}$ assumes local minimum/maximum values, $\mathbf{b}$ a slope with respect to a local maximum region (the red lines are the monotonic lines in the slope)
Fig. 6. a All slopes with respect to local minimum/maximum regions, $\mathbf{b}$ the smoothed image
$X_{i}$, let $v_{i}$ be the highest/lowest value assumed by pixels adjacent to $X_{i}$ if $\partial X_{i}$ is outward-falling/climbing. The smoothed image is a function $\tilde{f}: \Omega \rightarrow \mathbb{R}$ such that:

$$
\tilde{f}(x)= \begin{cases}v_{i} & \text { if } x \in X_{i} \text { for some } i \\ f(x) & \text { otherwise }\end{cases}
$$

Figure $6 \mathrm{~b}$ shows the smoothed image for our example. Let $f=\tilde{f}$ and $f_{E}$ be the extended function of $f$. Go to step (2).

After the reduced monotonic tree is generated, it is translated back to a square grid. For the example image given in Fig. 7a, its reduced monotonic tree is represented by its elements (nodes) at levels from 1-7, which are visually represented in Fig. 7b-h, respectively. In these pictures, an outwardfalling/climbing monotonic slope is shown by the white/black area which is enclosed by this slope. The blank area is shown in gray. The root node of the reduced monotonic tree of this example is at level 8 . The root node covers the whole image.

Compared with other hierarchical or multiscale models, such as a wavelet $[19,40]$, scale space [17], and peaks \& ridges [6], the monotonic tree model has the following advantages:

(1) The monotonic tree retrieves and represents the structures of an image at all scales. In addition, these structures are organized hierarchically as a tree, allowing easier analysis of the relationships between different levels.
(2) The monotonic tree retrieves the structures of an image directly and maintains their original shapes.

(3) Image structures are classified by their low-level features, then organized into clusters. Semantic features are drawn from the features of the clusters. The monotonic tree provides a vehicle for combining primitive features to characterize and capture semantic features.

\section{Extraction of semantic features}

The feature extraction scheme used here is based on reduced monotonic trees. We use the branches of the reduced monotonic tree to model the basic structures in an image, which are termed structural elements. Structural elements have lowlevel features such as color, shape, and spatial location. They are clustered to form high-level features.

Feature extraction consists of three consecutive steps:

(a) classifying structural elements;

(b) clustering structural elements; and

(c) rendering semantic regions.

Figure 8 shows the relationships among the steps given above.

The features of a given image are extracted and stored in a feature vector. A feature vector is an array which records the qualifying scores for all semantic features. The qualifying score of an image with respect to a feature indicates the degree 


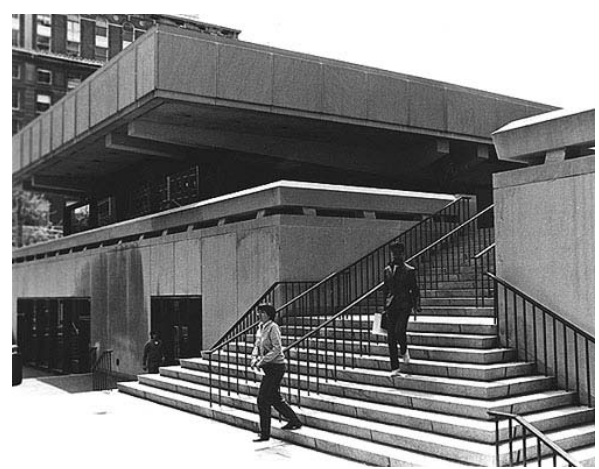

(a)

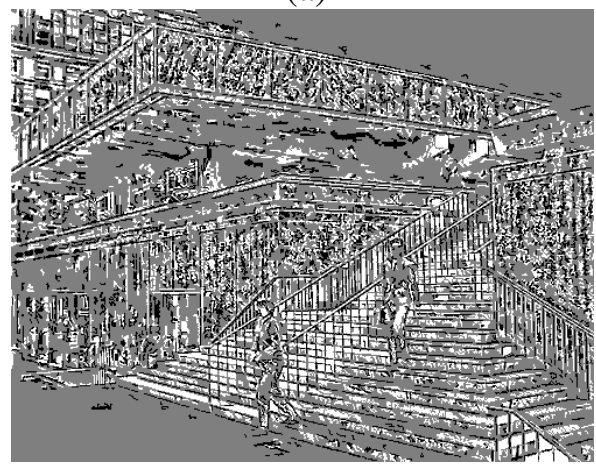

(c)

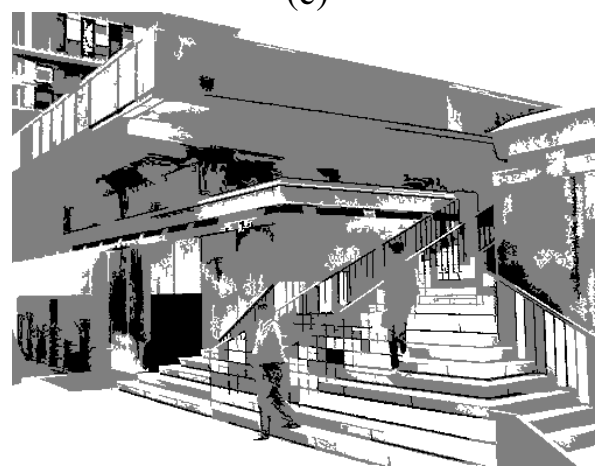

(e)

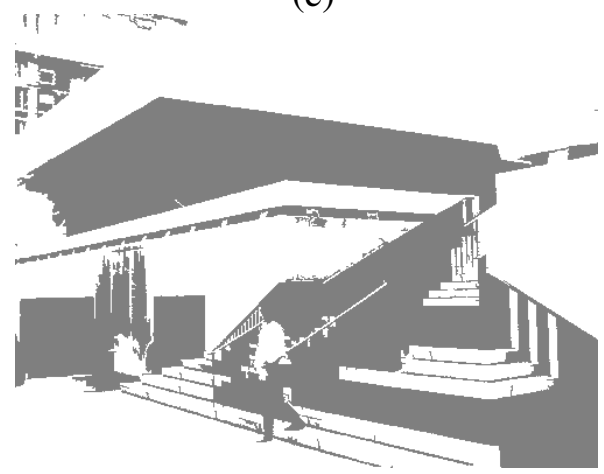

(g)

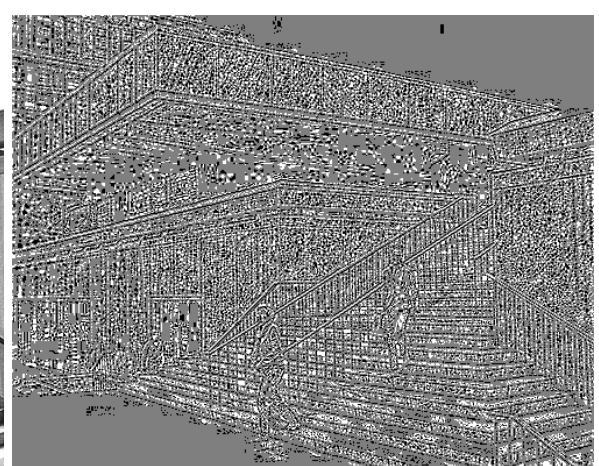

(b)

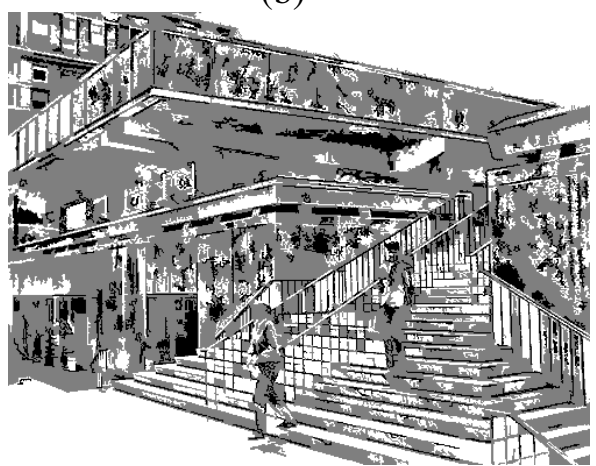

(d)

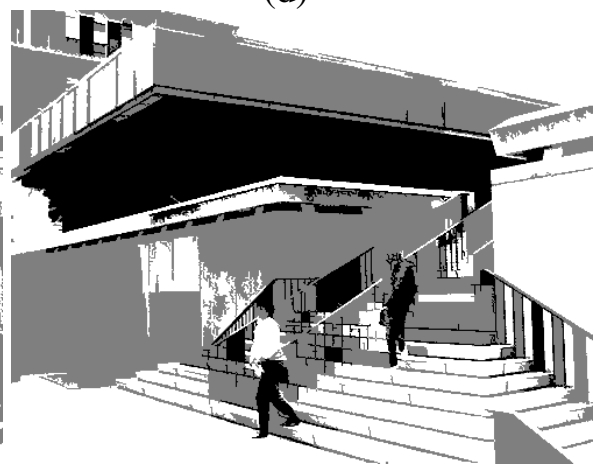

(f)

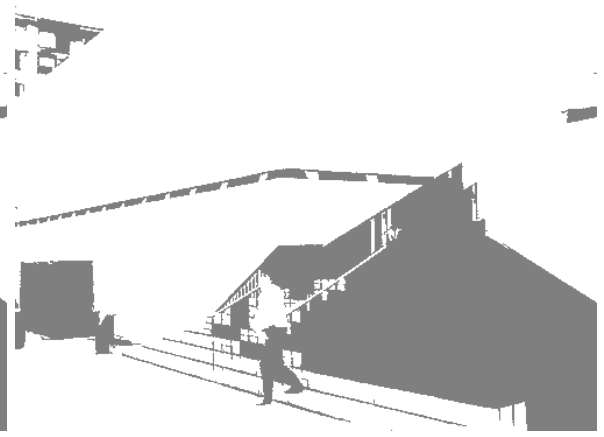

(h)
Fig. 7. An example of reduced monotonic tree 


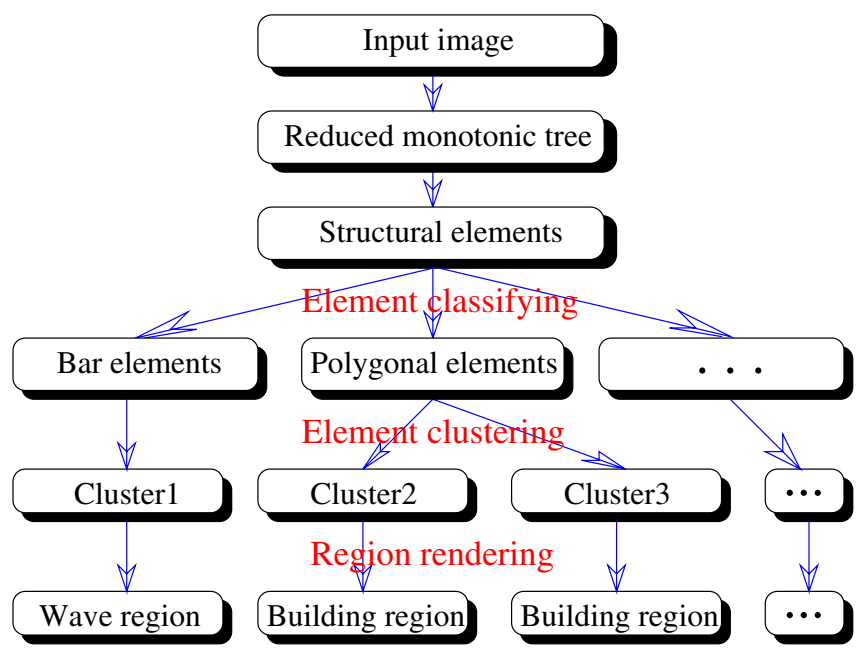

Fig. 8. System design for feature extraction

that the image can be considered to have this feature. Figure 9 illustrates the data structure of a feature vector.

\subsection{Classifying structural elements}

Each branch (or subtree) of a reduced monotonic tree is called a structural element if the image area it covers does not exceed an area threshold. A structural element is considered positive/negative if its root (i.e. the root of the subtree) is outward-falling/climbing, as shown in Fig. 10a. Positive/negative elements are like peaks/valleys. The altitude of a positive/negative element is defined as the absolute value of the average altitude of all its pixels above/below the highest/lowest pixels adjacent to the structural element, as shown in Fig. 10b. The harshness of a structural element is determined by the number, area, and altitude of its sub-elements. ${ }^{6}$ We define the harshness of an element $t$ by

$$
\begin{aligned}
& \text { Harshness }(t)= \\
& \frac{\Sigma_{b \in \text { SubElementSet }(t)} \text { Altitude }(b) * \text { Area }(b)}{\operatorname{Area}(t)},
\end{aligned}
$$

where SubElementSet $(t)$ is the set of sub-elements of $t$, Altitude $(b)$ is the altitude of $b$, and Area $(b)$ is the area of the region covered by $b$.

A structural element can be classified by its:

(1) color (the average color of all pixels in this element);

(2) altitude;

(3) harshness; and

(4) shape (the shape of its covered region).

For example, we can classify the structural elements by shape as:

(a) bars;

(b) polygons;

(c) irregular elements;

(d) smooth-boundary elements; and

\footnotetext{
${ }^{6}$ An element is a branch of the reduced monotonic tree. All subbranches of this branch are sub-elements.
}

(e) other shapes.

A bar element is characterized by its high length-towidth ratio, while a polygonal element is a structural element whose boundary consists mainly of line segments. A smoothboundary element has a boundary which forms a smooth curve. Irregular elements are those with irregular boundaries. Figure 11 shows structural elements of different shapes.

The semantic features of scenery images are characterized by categories of structural elements which are clustered in the images. Three examples of the categories are polygonal elements (for buildings), horizontal bar elements (for waves), and green harsh irregular elements (for trees), as shown in Figs. 12 and 13.

\subsection{Clustering structural elements}

Once the structural elements of an image have been classified, algorithms are applied to identify clusters of elements. First, the set of qualified (i.e. "belong-to") elements must be determined for each category. If two qualified elements overlap, the one with the lower qualifying score ${ }^{7}$ is eliminated from the category. This process is called element sifting. The sifting process reduces the multi-level elements of the image to single-level elements, all belonging to the given category. The elements after sifting form an element pattern in the 2D plane.

Next, as shown in Fig. 14, the Delaunay graph of the element pattern is constructed; this is the neighboring graph of the element pattern. We then apply clustering algorithms to the neighboring graph to find the clusters in the element pattern. Here, the clustering algorithm is based on the minimal spanning tree of the neighboring graph. Briefly, the process of clustering by minimal spanning tree is as follows. Let $V$ be the vertex set of the graph and $D$ be the distance threshold. Then $V$ is grouped into disjoint sets by joining all edges in the minimal spanning tree whose lengths (or weights) are less than or equal to $D$. Each set thus obtained is said to be a cluster at level $D$. Further background on pattern processing by neighboring graphs can be found elsewhere $[1,3]$. References on clustering by minimal spanning tree can be found in Zahn [49].

\subsection{Rendering semantic regions}

The process of rendering semantic regions from a cluster of structural elements consists of three steps: (1) elementconnecting; (2) hole-filling; and (3) boundary-smoothing. In the first step, all elements in the cluster are connected by line segments of lengths within a threshold. The second step involves filling all holes which are smaller than a given area threshold. Finally, the boundary of the region is smoothed by removing irregular angles and branches. These steps are illustrated in Fig. 15.

\footnotetext{
${ }^{7}$ For a given category, the qualifying score of an element indicates the degree that the element can be considered "qualified" to belong to this category. The process of arriving at a qualifying score will be discussed later.
} 


\begin{tabular}{|c|c|c|c|c|c|c|c|c|}
\hline \multirow[b]{2}{*}{ Features } & \multirow[b]{2}{*}{ Sky } & \multirow[b]{2}{*}{ Building } & \multirow[b]{2}{*}{ Tree } & \multirow[b]{2}{*}{ Wave } & \multirow[b]{2}{*}{$\begin{array}{l}\text { Placid } \\
\text { water }\end{array}$} & \multicolumn{3}{|c|}{ Ground } \\
\hline & & & & & & $\begin{array}{c}\ulcorner \\
\text { Lawn }\end{array}$ & $\begin{array}{c}1 \\
\text { Snow }\end{array}$ & Other \\
\hline Qualifying Scores & & & & & & & & \\
\hline
\end{tabular}

Fig. 9. Data structure of feature vector

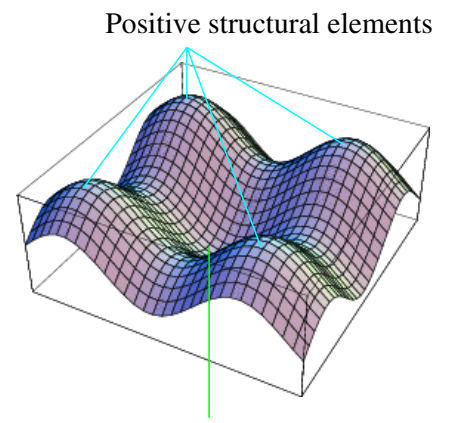

Negative structural element

a

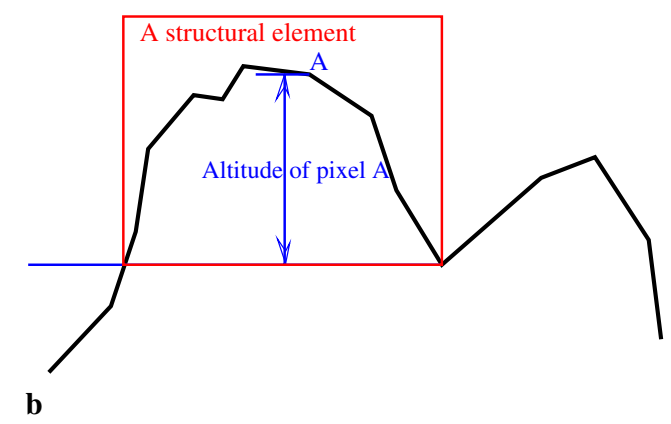

Fig. 10. a Positive/negative structural elements; $\mathbf{b}$ the altitude of a pixel in a structural element

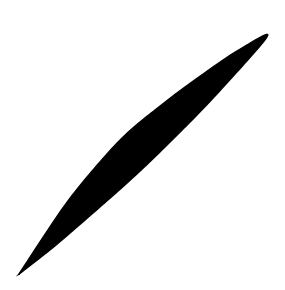

(a)

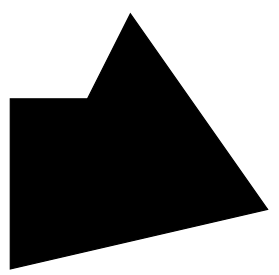

(b)

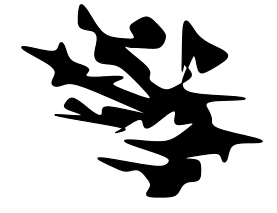

(c)

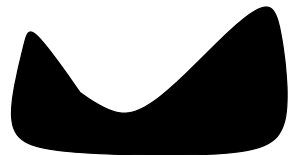

(d)

Fig. 11. a A bar element, b a polygonal element, $\mathbf{c}$ an irregular element, and $\mathbf{d}$ a smooth-boundary element

\subsection{Qualifying scores}

Qualifying scores must be established for structural elements, regions, and images. The qualifying score of a structural element measures the degree to which the element is considered to belong to a given category of elements. Qualifying scores for different element categories are determined in different ways. Formulas for computing the qualifying scores for various scenery-feature element categories will be presented in Sect. 4.

Similarly, the qualifying score of a region with a semantic feature measures the degree that the region manifests this particular feature. For a region generated from a cluster, its qualifying score is determined by the qualifying scores of the elements in the cluster and the area of the region. For example, a wave region is rendered from a cluster of horizontal bar elements. Let the input image be defined on the domain $\Omega$ and $R$ be a wave region generated from a cluster $S$ of horizontal bar elements. The qualifying score for $R$ with respect to wave is defined as

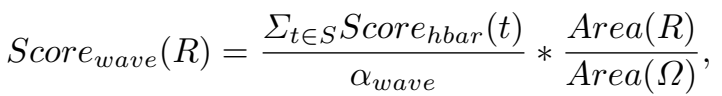

where $S_{\text {core }}$ hbar $(t)$ is the qualifying score for $t$ to be a horizontal bar element, and $\alpha_{\text {wave }}$ is a parameter to scale the qualifying score. $S$ core $_{\text {hbar }}(t)$ is defined in the next section.

In contrast, sky and ground regions are not generated from clusters. As will be discussed in the next section, we assume that these regions are smooth. Coarse regions are identified first; the smooth regions are then the complement of the coarse regions. The qualifying score of a smooth region is determined by the smoothness, color, and area of the region. As with the harshness of a structural element, the smoothness of a region is determined by the area and altitude of the structural elements intersecting this region:

$$
\begin{aligned}
& \text { Smoothness }(R)= \\
& \frac{\operatorname{Area}(R)}{\Sigma_{t \in \text { ElementSet }} \text { Altitude }(t) * \operatorname{Area}(\operatorname{Region}(t) \bigcap R)},
\end{aligned}
$$

where ElementSet is the set of all structural elements of the input image and Region $(t)$ is the region covered by $t$. The qualifying score for a region $R$ to be considered a sky region is defined as ${ }^{8}$

$$
\begin{aligned}
& \operatorname{Score}_{\text {sky }}(R)= \\
& \frac{\operatorname{Smoothness}_{(R)}}{\alpha_{\text {sky }}} * \frac{\operatorname{Area}(R)}{\operatorname{Area}(\Omega)} * \operatorname{Sky} \operatorname{Color} \operatorname{Ratio}(R),
\end{aligned}
$$

where $\alpha_{s k y}$ is a parameter to scale the qualifying score and SkyColorRatio $(R)$ is the ratio of sky-colored pixels in $R .{ }^{9}$ The qualifying scores for ground and placid-water regions are defined similarly.

\footnotetext{
${ }^{8}$ Region $R$ should first be examined to ascertain that it satisfies our assumptions about sky regions.

${ }^{9}$ We assume that the sky color is either blue or gray (the color of clouds). These assumptions will be discussed in the next section.
} 

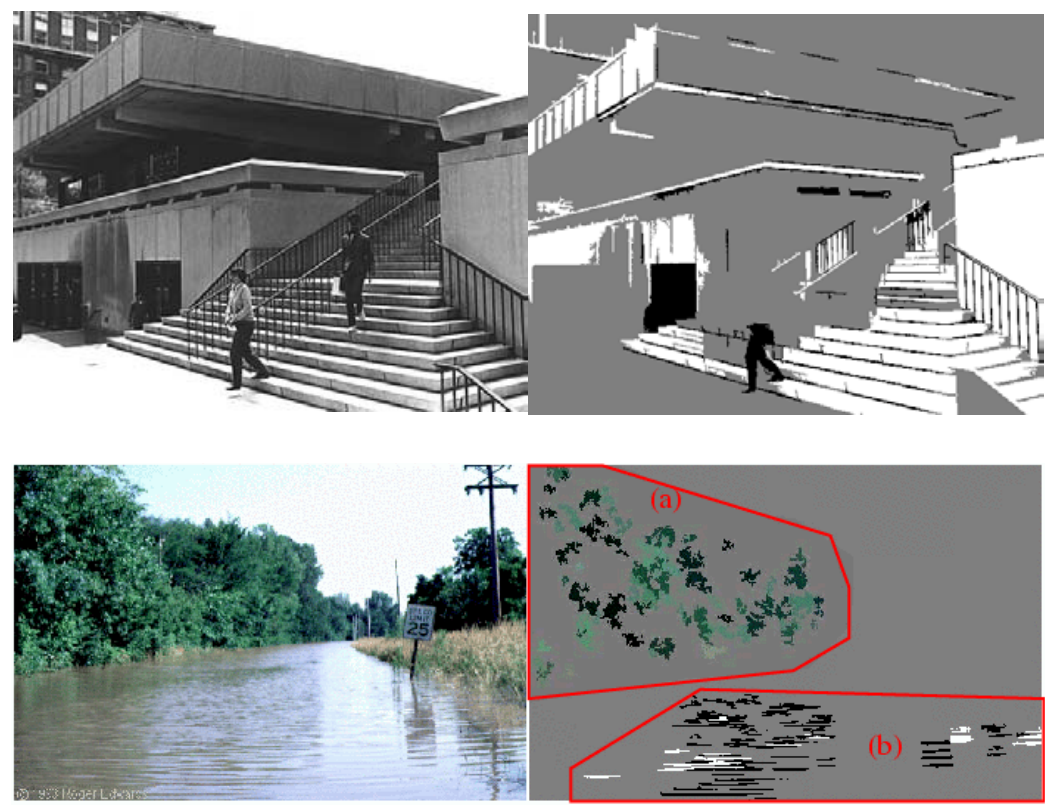

Fig. 12. An image and its polygonal elements (shown in black and white)

Fig. 13. An image and its green harsh irregular elements (shown in green or dark green) as well as its horizontal bar elements (shown in black and white)

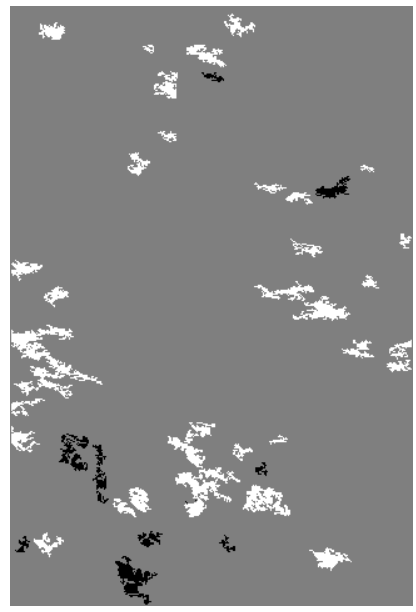

a

Fig. 14. a An element pattern, and $\mathbf{b}$ the neighboring graph of the pattern

An input image may have several regions which manifest a specific feature. The qualifying score for the input image with respect to this feature is defined as the sum of qualifying scores of these regions. For example, let $I$ be the input image and $R_{1}, R_{2}, \ldots, R_{n}$ be the tree regions found in this image. The qualifying score of $I$ with respect to "tree" is

Score $_{\text {tree }}(I)=\Sigma_{i=1}^{n} \operatorname{Score}_{\text {tree }}\left(R_{i}\right)$.

If an input image contains no regions which manifest a specific feature, then the qualifying score for the image with respect to this feature is zero.

\section{Case study of semantic features}

The previous section described a general scheme for the extraction of semantic features. In this section, we present meth-

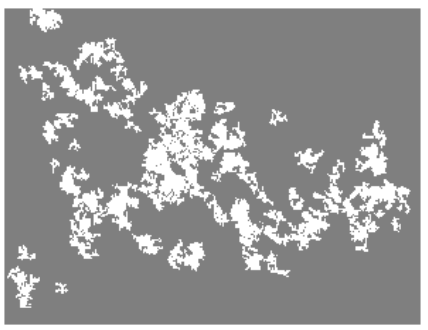

(a)

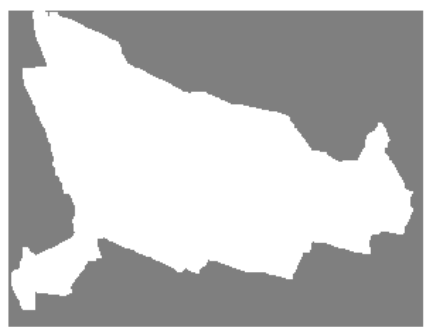

(c)

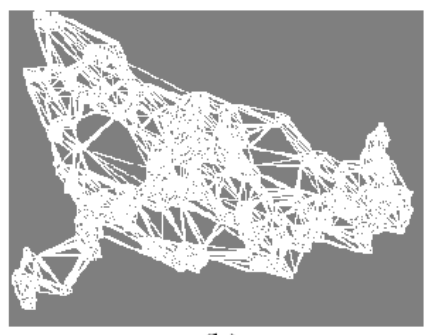

(b)

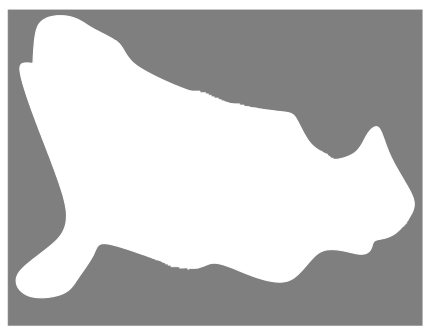

(d)
Fig. 15. a A cluster of structural elements, b element connecting, c hole filling, and $\mathbf{d}$ boundary smoothing

ods for defining the categories of structural elements and identifying the regions with scenery features.

The following discussion is predicated upon some simple assumptions about the colors of scenery features. For example, trees are assumed to be green and water to be blue. More comprehensive color patterns for scenery features can be integrated into our system, though this is not the focus of this paper.

We first briefly introduce our approach to color modeling. A pixel is a gray pixel if none of its red, green, and blue components is dominant, i.e.

$$
\max (r, g, b)-\min (r, g, b) \leq \mathcal{T}^{g},
$$

where $r, g$, and $b$ are the red, green, and blue components of the pixel, respectively, and $\mathcal{T}^{g}$ is a threshold. A pixel is red, 
green, or blue if

$$
\begin{aligned}
r>\max (g, b) & +\mathcal{T}^{g}, g>\max (r, b) \\
& +\mathcal{T}^{g}, \text { or } b>\max (r, g)+\mathcal{T}^{g},
\end{aligned}
$$

respectively. A pixel is white if it is gray and

$$
(3 * r+10 * g+b) / 10 \geq \mathcal{T}^{w},
$$

where $(3 * r+10 * g+b) / 10$ is the brightness of the pixel and $\mathcal{T}^{w}$ is a threshold. A region is red (green, blue, gray, or white) if the ratio of red (green, blue, gray, or white) pixels in the region is greater than or equal to a threshold.

We also make assumptions about the location of sky and ground regions in images. For a region $R$ in an image $I$, its location is modeled by its top, bottom, left, and right positions: ${ }^{10}$

$$
\begin{aligned}
\operatorname{TopPos}(R) & =\min \{y \mid \exists x,(x, y) \in R\}, \\
\text { BottomPos }(R) & =\max \{y \mid \exists x,(x, y) \in R\}, \\
\text { LeftPos }(R) & =\min \{x \mid \exists y,(x, y) \in R\}, \\
\text { RightPos }(R) & =\max \{x \mid \exists y,(x, y) \in R\} .
\end{aligned}
$$

Suppose the domain of image $I$ is $\{0,1, \ldots, N-1\} \times$ $\{0,1, \ldots, M-1\}$. We say that a region $R$ is in the lower part of the image if $\frac{\operatorname{Top} P o s(R)}{M}$ is greater than a threshold; we say that a region occupies an upper part of the image if $\frac{\operatorname{Top} P o s(R)}{M}$ is less than a threshold.

\subsection{Sky}

The sky is a kind of background. Without clouds, a sky region is a homogeneous region consisting of blue pixels. Due to their physical properties, clouds in an image tend to change smoothly at the pixel level. From a location's stand point, there is usually no other object above a sky region. To retrieve sky regions, we make three simple assumptions:

- (sky.a1) a sky region is smooth;

- (sky.a2) a sky region occupies an upper part of the image; and

- (sky.a3) the color of sky regions is either blue or the color of clouds.

For our current implementation, we assume that the color of clouds is gray. To find the sky regions, we first identify the smooth regions in the image. The smooth regions are the complement of the harsh regions, which are characterized by peaks and valleys in intensity. Within the monotonic tree, these peaks and valleys are modeled as small structural elements with high altitudes. Thus, the harsh regions of the image are detected by clustering these small, high-altitude elements, as discussed in the last section. Identification of the harsh regions automatically distinguishes the smooth regions as well. These smooth regions are then checked for location and color to find the sky regions.

\footnotetext{
${ }^{10}$ Here, we assume the monitor coordinate system: the $x$-axis is pointing right and the $y$-axis is pointing down.
}

\subsection{Ground and placid water}

We make three assumptions about ground regions:

- (ground.a1) a ground region is smooth;

- (ground.a2) a ground region is in the lower part of the image; and

- (ground.a3) in a ground region, structural elements are more horizontal than vertical.

When scenery images are taken, the direction of the projection is usually horizontal or nearly horizontal. Thus, as the natural scene is projected onto the image plane, ground-level structures appear more horizontal than vertical, leading to the third assumption above.

Let us first discuss methods for modeling the horizontality of a structural element. For a region $X$ on the square grid $\mathbb{Z}^{2}$ and some $x_{0} \in \mathbb{Z}$, we define the height of $X$ at $x_{0}$ to be the number of pixels in the intersection between $X$ and the vertical line crossing $\left(x_{0}, 0\right)$. Formally,

$$
\operatorname{Height}\left(X, x_{0}\right)=\left|\left\{\left(x_{0}, y\right) \mid\left(x_{0}, y\right) \in X\right\}\right|,
$$

where $|\bullet|$ gives the number of elements in a set. Similarly, we define the width of $X$ at $y_{0}$ as

$$
\operatorname{Width}\left(X, y_{0}\right)=\left|\left\{\left(x, y_{0}\right) \mid\left(x, y_{0}\right) \in X\right\}\right| .
$$

Now we define the horizontal and vertical ratios of $X$ to be

$$
\begin{aligned}
& \text { HorizontalRatio }(X)= \\
& \sqrt{\frac{\Sigma_{y \in \mathbb{Z}}(\operatorname{Width}(X, y))^{2}}{\Sigma_{x \in \mathbb{Z}}(\operatorname{Height}(X, x))^{2}}} ; \text { and } \\
& \operatorname{VerticalRatio}(X)=\frac{1}{\text { HorizontalRatio }(X)} .
\end{aligned}
$$

For a rectangle $\{0,1, \ldots, N-1\} \times\{0,1, \ldots, M-1\}$, its horizontal ratio is $\frac{N}{M}$. A structural element is said to be horizontal (or vertical) if the horizontal (or vertical) ratio of its covered region is greater than a given threshold.

As with the detection of sky regions, the identification of ground and placid-water regions starts with finding the smooth regions in the image. Then, for each smooth region, we check the validity of assumptions (ground.a2) and (ground.a3). To test (ground.a3), we count the horizontal and vertical elements in the smooth region. The last assumption holds if the horizontal elements exceed the vertical elements in the region.

A ground region identified by this method could be lawn, snow, or other ground subtypes. These region subtypes are distinguished by color. For example, we assume that lawn regions are green and snow regions are white.

Identification of placid-water regions is based upon four assumptions. The first three are the same as those used for ground identification. In addition, we assume that the color of placid water is blue.

\subsection{Wave}

Small water waves are characterized by very regular patterns which appear as horizontal bars in a horizontally-projected 

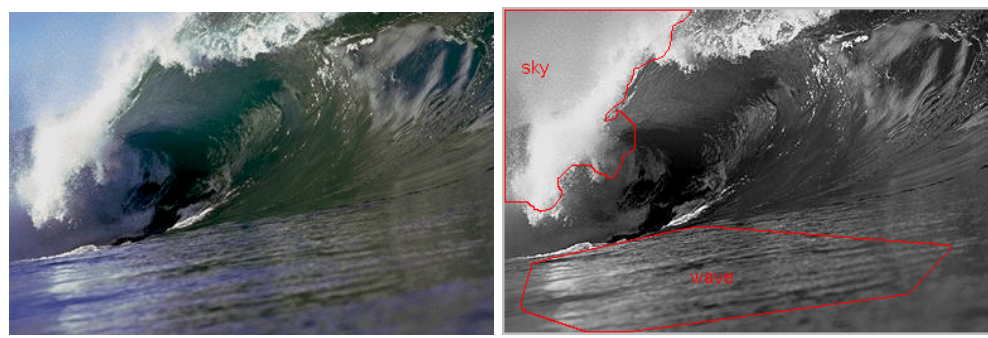

Fig. 16. An example of an image with breaking waves and its annotation

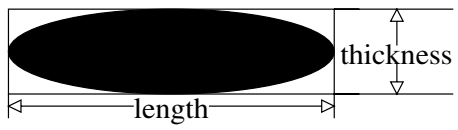

BarRatio $=$ length/thickness

Fig. 17. The bar ratio of an ellipse

image, such as that shown in Fig. 13. Breaking waves have complicated structures. However, images with breaking waves usually contain an area of water surface which consists of parallel bar structures, as in the image shown in Fig. 16.

We define a wave region as a region consisting of horizontal bar elements. A bar is characterized by its high length-tothickness ratio. For a $2 \mathrm{D}$ region $X$, we define its length and thickness as

$$
\begin{array}{r}
\operatorname{Length}(X)=\max _{x, y \in X}\|X-Y\|, \\
\operatorname{Thickness}(X)=\min _{v \in \mathbb{R}^{2}} \max _{x, y \in X} \frac{(x-y) \bullet v}{\|v\|},
\end{array}
$$

respectively, where $(x-y) \bullet v$ is the inner product of vectors $x-y$ and $v$, and $\|v\|$ is the length of the vector $v$. We define the bar ratio of a region $X$ as

$$
\operatorname{BarRatio}(X)=\frac{\operatorname{Length}(X)}{\text { Thickness }(X)} .
$$

This situation is illustrated in Fig. 17. A structural element is considered to be a bar element if the bar ratio of its covered region is higher than a given threshold. The qualifying score of a structural element to be a horizontal bar element is defined as the product of the bar ratio and horizontal ratio of its covered region:

$$
\begin{aligned}
\operatorname{Score}_{\text {hbar }}(t) & =\operatorname{BarRatio}(\operatorname{Region}(t)) \\
& * \text { HorizontalRatio }(\text { Region }(t)) .
\end{aligned}
$$

Wave regions are identified by clustering horizontal bar elements in the image.

\subsection{Green tree}

A careful examination of the tree region in Fig. 13 reveals that the micro-structures in the region are very irregular. Based on this observation, we assume that a tree region is a region consisting of green harsh irregular elements. The tree regions in an image are found by clustering the green harsh irregular elements in the image.

For a connected region $X$, let ConvexHullLength $(X)$ be the length of its convex hull and $\operatorname{Length}(\partial X)$ be the length of its boundary. We define the irregularity of $X$ by

$$
\operatorname{Irregularity}(X)=\frac{\operatorname{Length}(\partial X)}{\text { ConvexHullLength }(X)} \text {. }
$$

The qualifying score of a structural element to be a tree element is defined as the product of its harshness and the irregularity of its covered region:

$$
\begin{aligned}
\text { Score }_{\text {treeElement }}(t) & =\text { Harshness }(t) \\
& * \operatorname{Irregularity}(\operatorname{Region}(t)) .
\end{aligned}
$$

\subsection{Building}

The shapes of most buildings are characterized by the line segments they contain. We assume that a building region in an image is a region consisting of polygonal elements. To ascertain whether a structural element is polygonal, we first partition its boundary into line segments and other kinds of segments. A structural element is polygonal if its boundary mainly consists of line segments. References on curve partitioning can be found elsewhere [29-31].

For a connected region $X$, let $L(\partial X)$ be the length of its boundary and $L L S(\partial X)$ be the total length of the line segments detected in its boundary. We define the polygon ratio of $X$ by

$$
\operatorname{PolygonRatio}(X)=\frac{L L S(\partial X)}{L(\partial X)} \text {. }
$$

The qualifying score of a structural element to be a polygonal element is defined as the polygon ratio of its covered region.

\section{Experiments}

Comprehensive experiments were conducted to demonstrate the effectiveness of the proposed approach; these experiments employed our SceneryAnalyzer system. ${ }^{11}$ First, performance comparisons using COREL images were made between the proposed approach and some traditional CBIR techniques. These comparisons were based on the precision-recall for individual scenery features. We then conducted experiments using PhotoDisc images to verify that the performance of our approach is not source-sensitive. Finally, the proposed approach was applied to both COREL and PhotoDisc images to explore its effectiveness with different combinations of scenery features. Examples of some of these images and their copies annotated by SceneryAnalyzer are provided at the end of this section.

\footnotetext{
11 The system can be accessed at http://monet.cse.buffalo.edu: $8888 /$.
} 


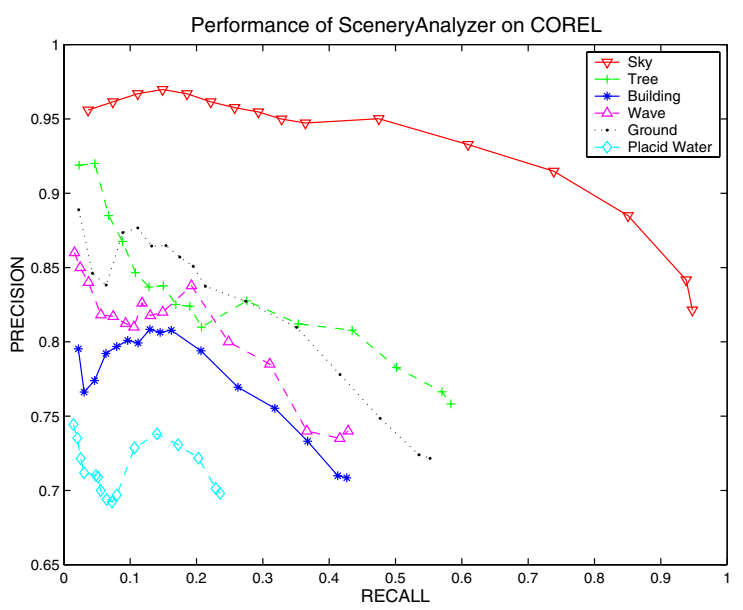

(a)

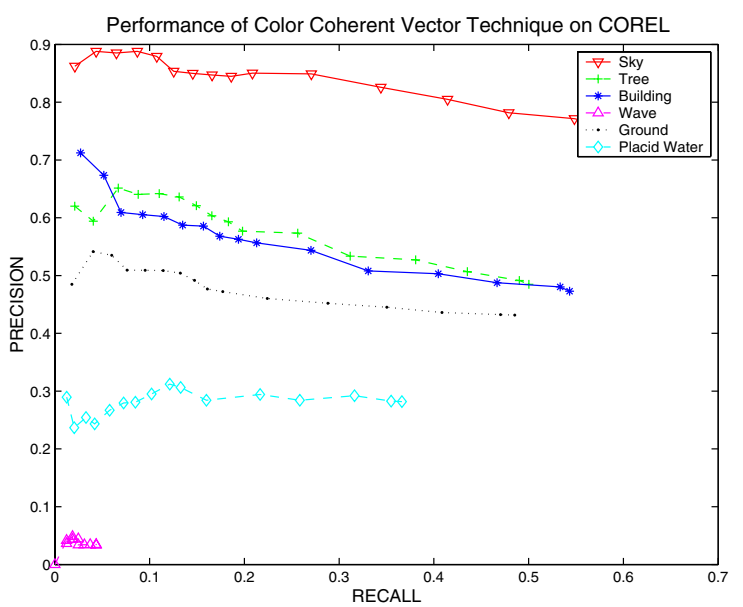

(c)

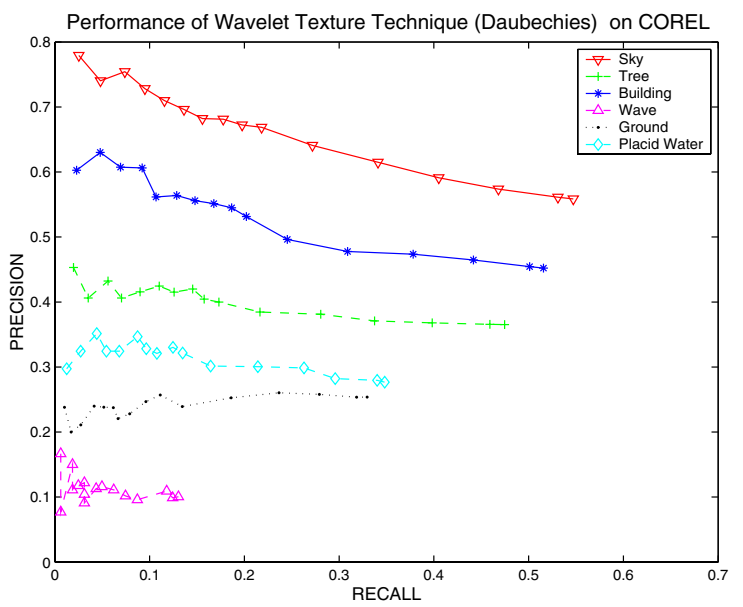

(e)

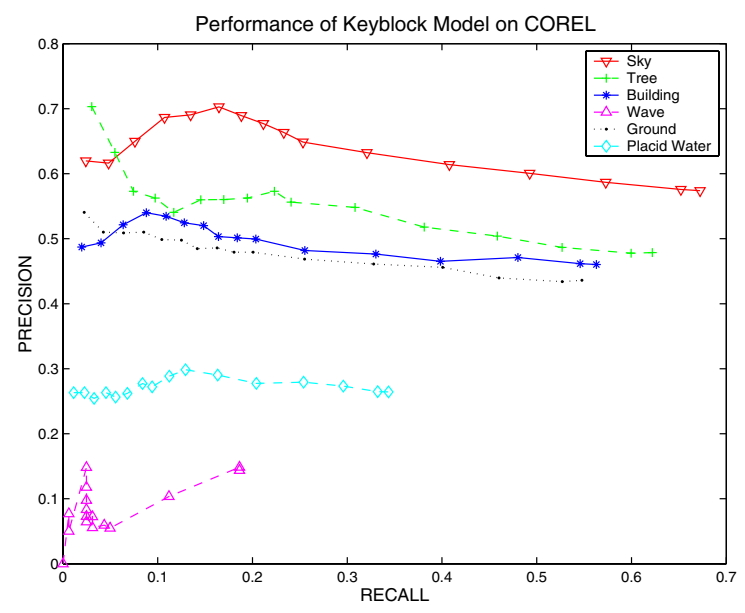

(b)

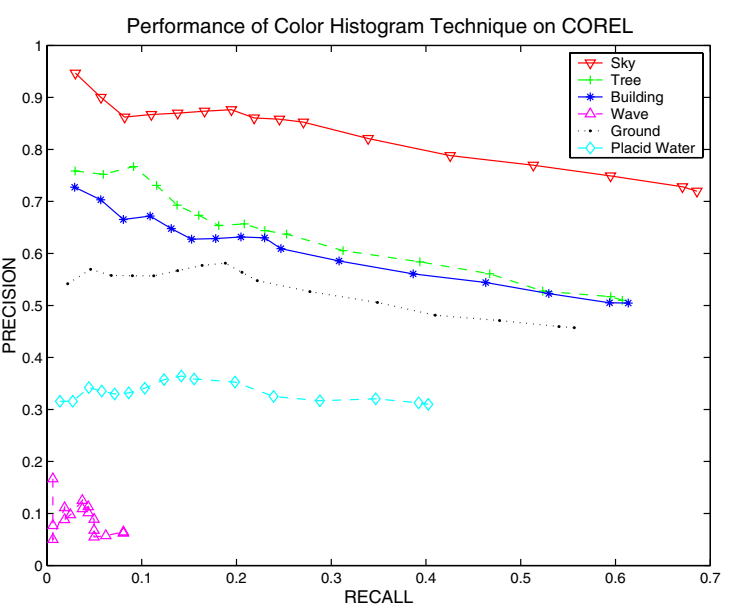

(d)

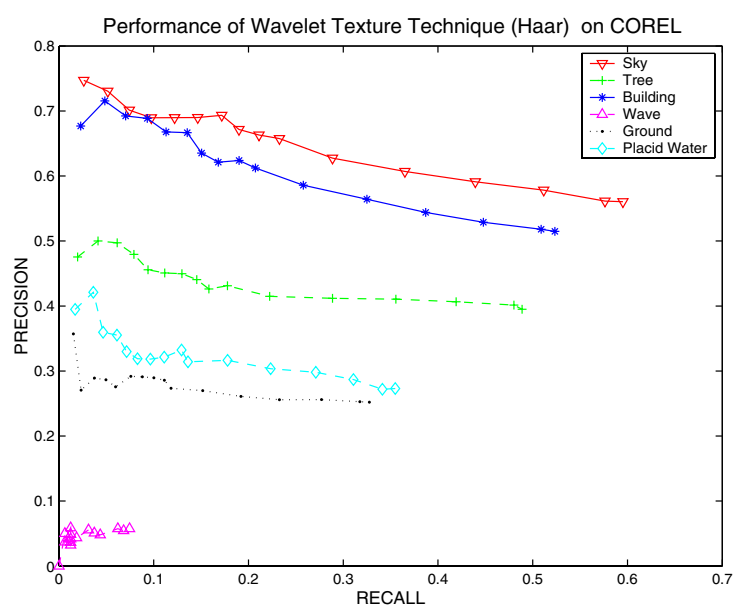

(f)

Fig. 18. Performance of a SceneryAnalyzer, $\mathbf{b}$ keyblock model, $\mathbf{c}$ color coherent vector, $\mathbf{d}$ color histogram, $\mathbf{e}$ Daubechies wavelet, and $\mathbf{f}$ Haar wavelet on COREL images 


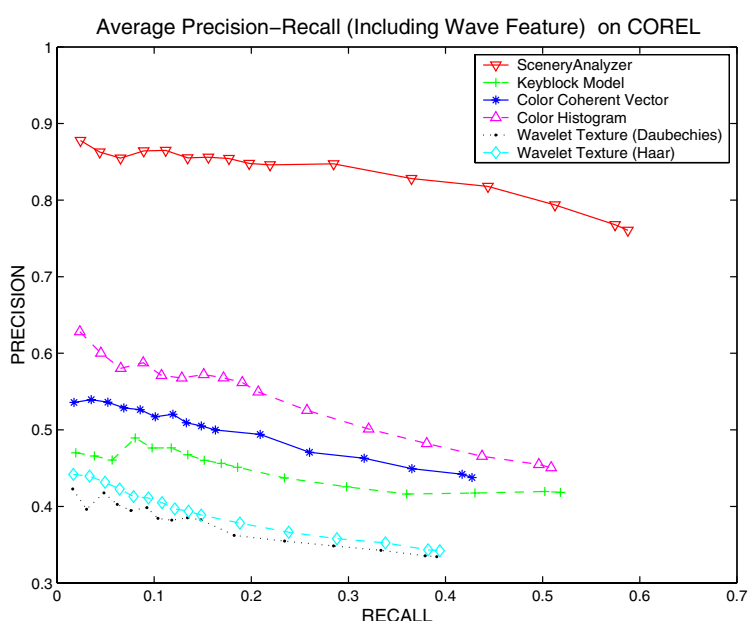

a

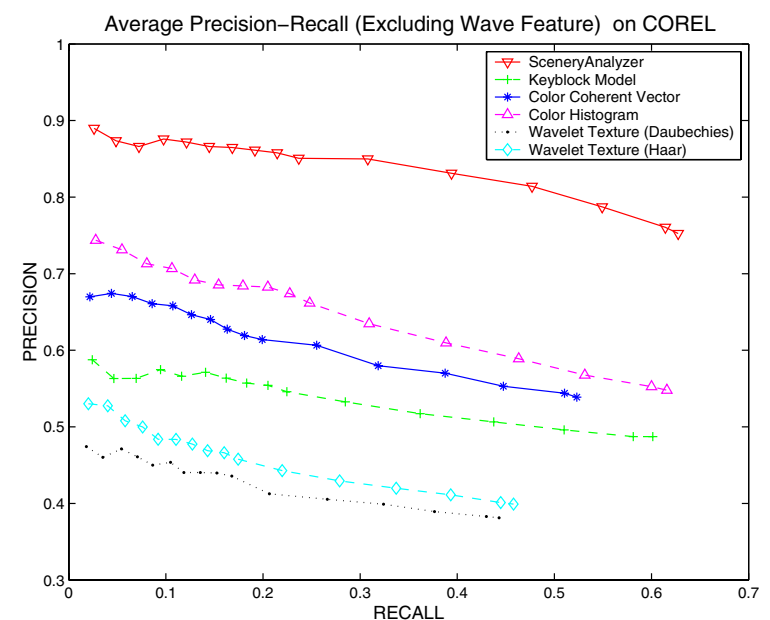

b

Fig. 19. a Average precision-recall for six features: sky, building, tree, wave, ground, and placid water; b average precision-recall for five features: sky, building, tree, ground, and placid water

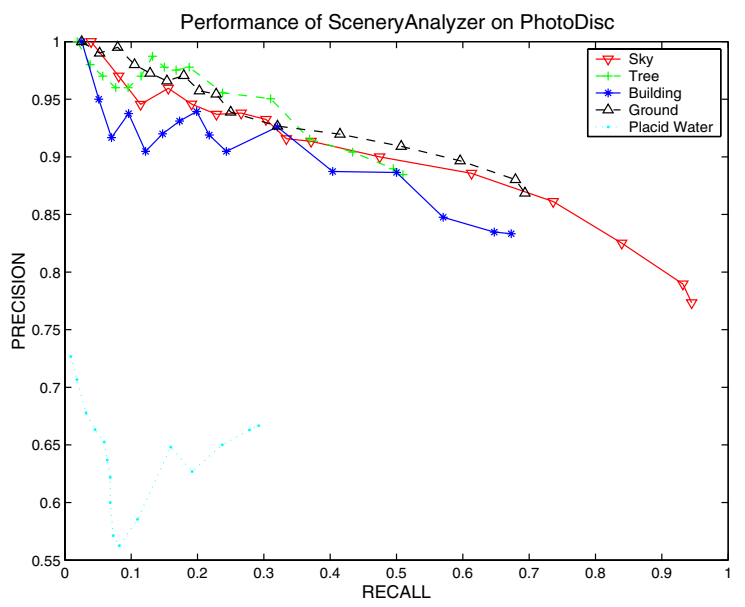

(a)

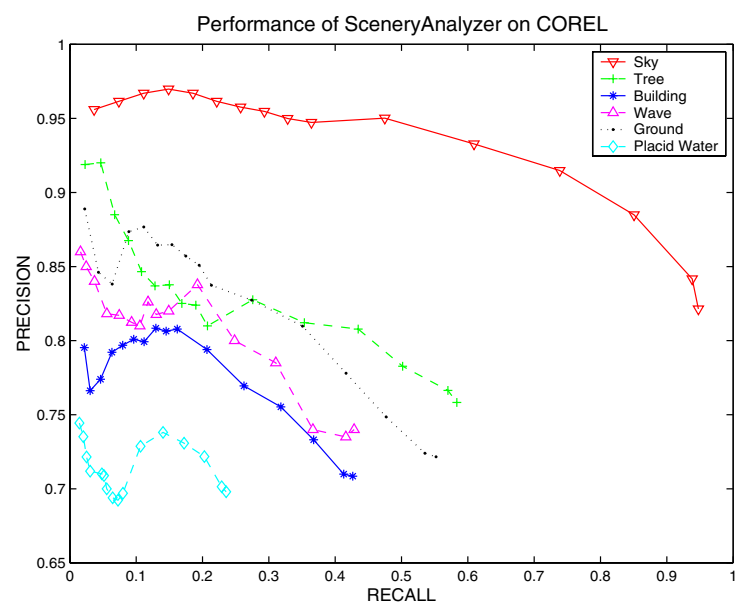

(c)

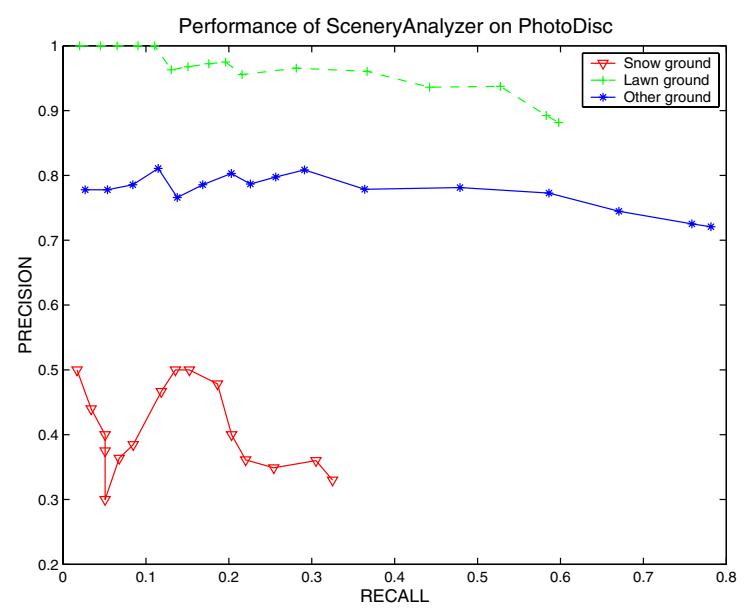

(b)

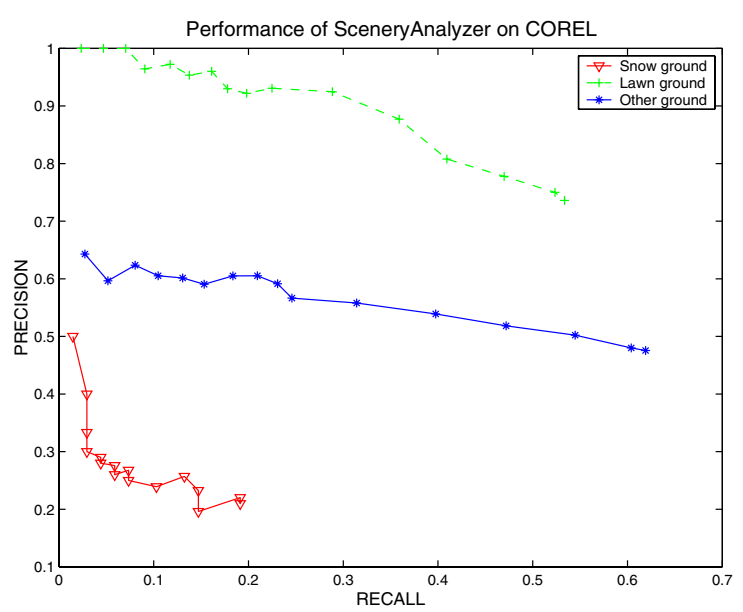

(d)

Fig. 20. a Performance of SceneryAnalyzer on PhotoDisc database with respect to sky, building, tree, placid water, and ground; $\mathbf{b}$ performance of SceneryAnalyzer on PhotoDisc database with respect to snow, water, lawn, and other kind of ground; c performance of SceneryAnalyzer on COREL database with respect to sky, building, tree, wave, placid water, and ground; $\mathbf{d}$ performance of SceneryAnalyzer on COREL database with respect to snow, lawn, and other kind of ground 

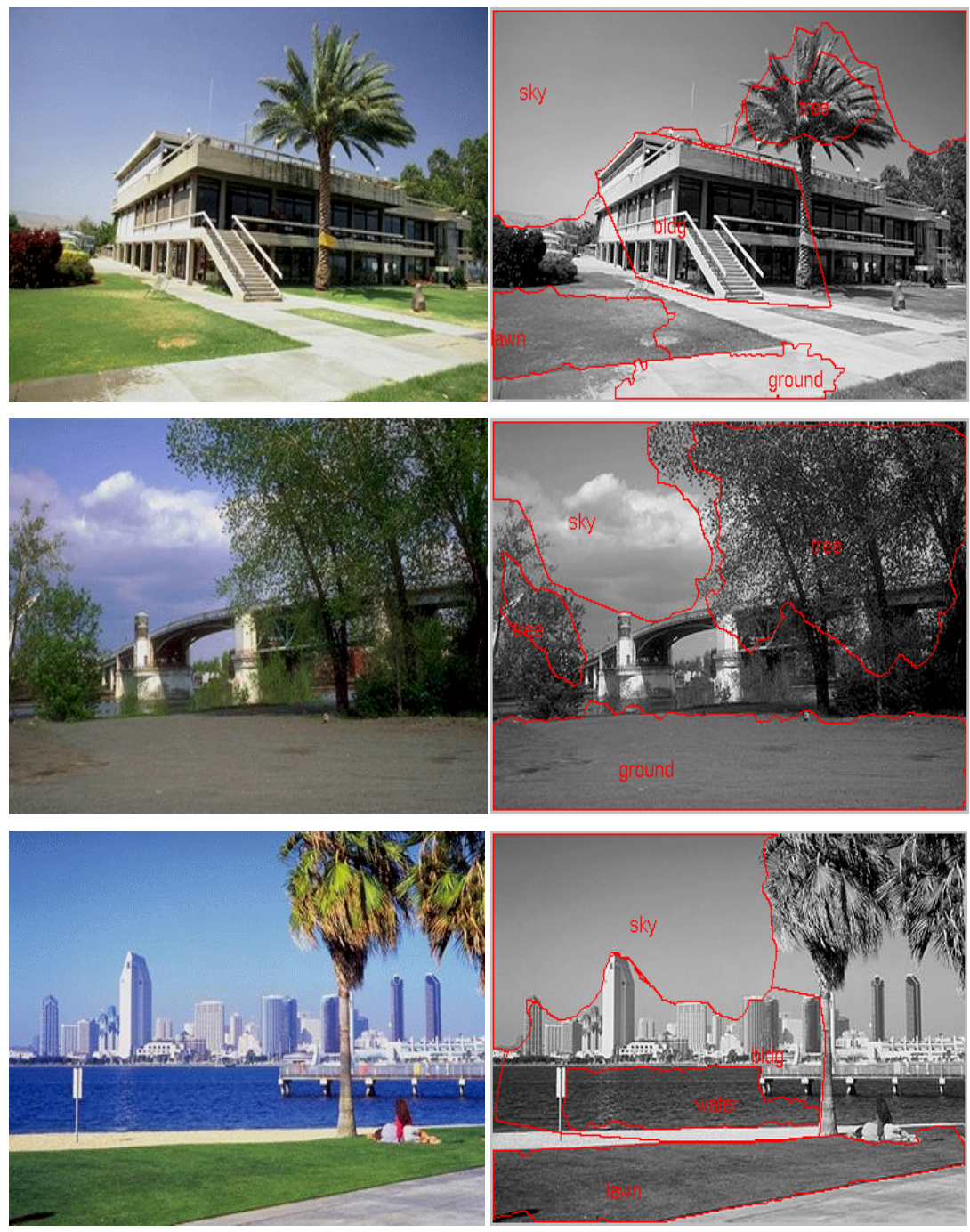

Fig. 21. Some examples of scenery images and their annotated copies

\subsection{Experiment setup}

In our implementation, we used many parameters for extracting features from images. These parameters were selected manually: we tested the system with about 100 images from different sources and with different types of scenery features, and selected the parameters such that the system had the best performance on these images. In future work, machine learning techniques will be used for automatic optimization of parameters. The system was implemented in a Sun Ultra 10. For an image of size $384 \times 256$, an average time for off-line processing is about two minutes.

Two image databases were employed in these experiments. The first image database, COREL, consists of 6776 color images from CD7 and CD8 of COREL Gallery 1,300,000. These photos are stored in JPEG format and are sized at either $256 \times 384$ or $384 \times 256$. The COREL database includes both scenery and non-scenery images. There are 4125 scenery pictures taken at globally diverse locations, along with 2651 nonscenery images covering a wide variety of subjects, including fish, molecules, space scenes, insects, and other topics. The second image database, PhotoDisc, consists of 1444 images from PhotoDisc Comping Discs 3 and 4. These images fall into five categories: (1) homes and gardens, (2) international sports, (3) nature scenes, (4) panoramic landscapes, and (5) people, lifestyles, and vacations. Table 1 shows a breakdown of these two image databases by scenery features.

For each scenery feature, the precision-recall of SceneryAnalyzer was calculated and plotted. Let RETRIEVELIST be the list of $n$ images retrieved with a given feature, which is sorted in descending order by qualifying scores. We calculate the precision and recall for each of the first $\frac{n}{30}, \frac{2 n}{30}, \frac{3 n}{30}, \ldots$, and $n$ images in RETRIEVELIST. The precision and recall figures were then plotted to demonstrate the performance of SceneryAnalyzer.

In Sect. 5.2, performance with the COREL database will be used to compare SceneryAnalyzer with the keyblock model [16], traditional color histogram [42], color coherent vector [26], and wavelet (Daubechies and Haar) texture techniques $[36,40]$. All these methods accept only queries by examples. The process of selecting query sets and calculating the precision-recall for these methods can be illustrated with a typical case, where the sky feature is queried using the keyblock approach. There are 2369 COREL images with sky regions. Each sky image is used as a keyblock query on the COREL database; the top 100 images are thus determined, and the number of sky images in this retrieved set is counted. The 

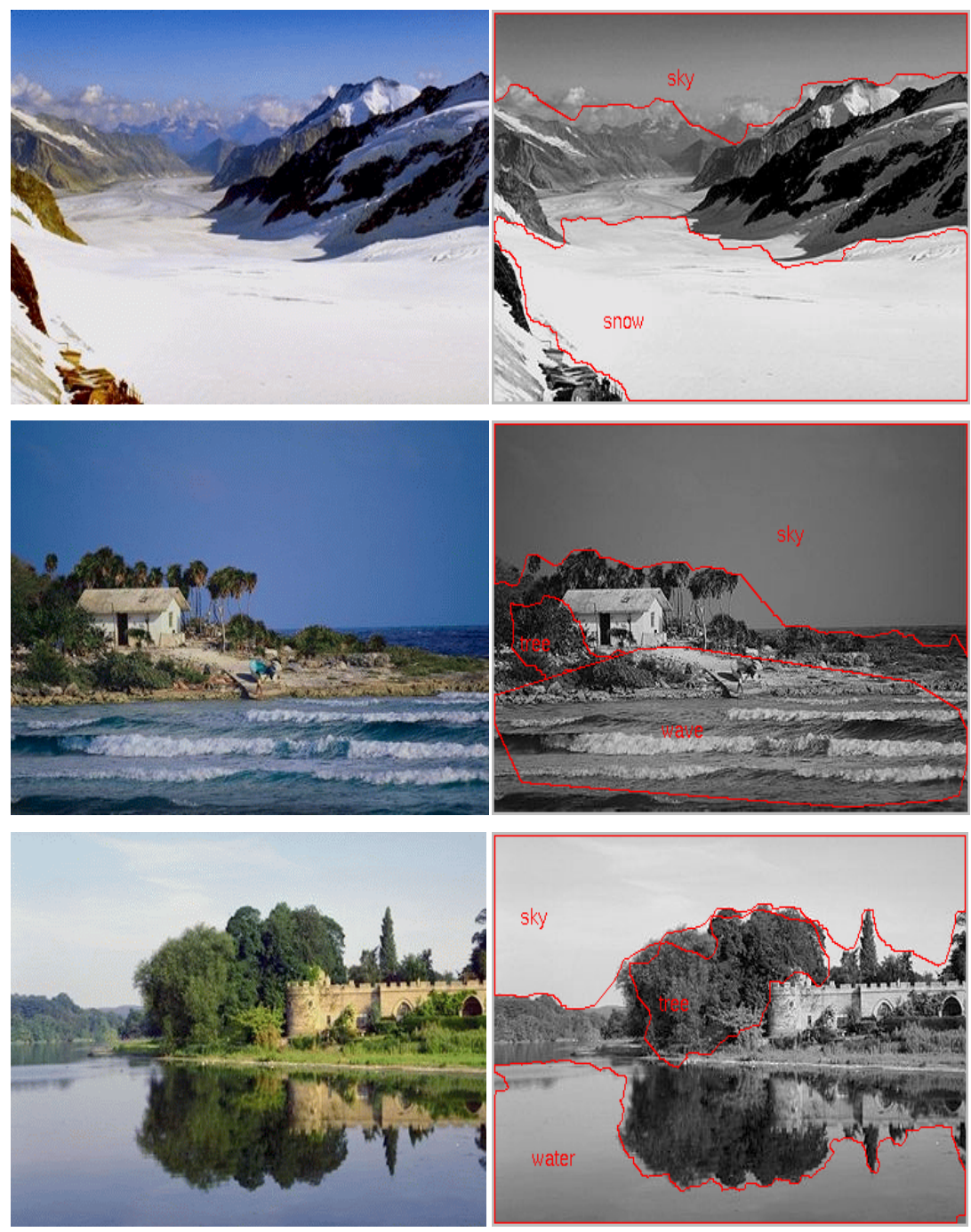

Fig. 22. Some examples of scenery images and their annotated copies

Table 1. Breakdown by scenery features of images in COREL and PhotoDisc databases

\begin{tabular}{|c|c|c|c|c|c|c|c|c|}
\hline \multirow[t]{2}{*}{ Feature } & \multirow[t]{2}{*}{ Sky } & \multirow[t]{2}{*}{ Building } & \multirow[t]{2}{*}{ Tree } & \multirow[t]{2}{*}{ Wave } & \multirow{2}{*}{$\begin{array}{l}\text { Placid } \\
\text { Water }\end{array}$} & \multicolumn{3}{|c|}{ ground } \\
\hline & & & & & & Lawn & Snow & Other \\
\hline $\begin{array}{l}\text { COREL images } \\
\text { with the feature } \\
\text { PhotoDisc images }\end{array}$ & 2369 & 1920 & 1479 & 161 & 882 & 298 & 68 & 659 \\
\hline with the feature & 455 & 156 & 382 & 6 & 219 & 57 & 59 & 261 \\
\hline
\end{tabular}

2369 sky images are then sorted in descending order by the number of sky images in their corresponding retrieved sets. The sorted list is denoted as SKYLIST. The first 5\% (i.e. 118 of images from SKYLIST) are then selected as the query set and denoted as QUERYSET. For each COREL image $I$, we calculate its distance to QUERYSET- $\{I\}$ using the keyblock approach. ${ }^{12,13}$ The COREL images are then sorted in ascending order by this distance. The top 2369 (i.e. the number of

\footnotetext{
12 The distance from an image to a set of images is the shortest distance from this image to the images in the set.

${ }^{13}$ If we retrieve by the distances of images to QUERYSET, the images in QUERYSET will have distance zero. Thus, the query images will automatically be retrieved as the top $5 \%$ images, which provides an unfair comparison.
}

images with sky) COREL images are retrieved. Using the images retrieved by the keyblock model, we then calculate and plot the precision-recall of the keyblock model for the sky feature.

Section 5.4 compares the performance of SceneryAnalyzer with the traditional CBIR techniques on combinations of scenery features. For this comparison, these techniques are used to retrieve the top 20 images which have the specified combination of scenery features. Query sets are selected and images retrieved in the manner described above. 


\subsection{Comparison using COREL database}

Experiments were conducted using 6776 COREL images to compare SceneryAnalyzer with the keyblock approach [16], traditional color histogram [42], color coherent vector [26], and wavelet (Haar and Daubechies) texture techniques [36, 40]. The precision-recall of these techniques was compared for six scenery features: sky, building, tree, wave, ground, and placid water.

The precision-recall of each method was calculated for each scenery feature listed; these results appear in Fig. 18. The five traditional CBIR methods all performed poorly with the wave feature. This can be partially explained by the small number of wave images in the COREL database, which makes these methods easily misidentify other images as wave images. Furthermore, these methods did not capture the structures of waves.

A comparison of the graphs in Fig. 18 shows that our method outperforms all the others for each scenery feature type. To further clarify the comparison, we calculated the average precision-recall over all six scenery features, which is shown in Fig. 19a. The average precision-recall of the five traditional methods suffers particularly from their poor performance with the wave feature, as discussed above. To exclude this effect, we also calculated the average precision-recall over the five remaining scenery feature types (sky, building, tree, ground, and placid water); this is shown in Fig. 19b. These comparisons indicate that our proposed method provides much better precision-recall of scenery features than the traditional techniques.

\subsection{Experiments using PhotoDisc database}

We also conducted experiments on 1444 PhotoDisc images. The performance of SceneryAnalyzer with this PhotoDisc database is shown in Fig. 20a and b. Since the PhotoDisc database included only six images with waves, a precisionrecall curve could not be plotted for the wave feature. Thus, Fig. 20a shows only five precision-recall curves. It is worth noting, however, that when queried for wave images on this database, SceneryAnalyzer retrieved three images, all with waves. Therefore, the precision of these queries was $100 \%$, with a $50 \%$ recall. As a comparison, the performance of SceneryAnalyzer with the COREL database is shown in Fig. 20c and d. These experiments show that SceneryAnalyzer works effectively with both COREL and PhotoDisc images.

With both the COREL and PhotoDisc databases, the performance of SceneryAnalyzer is relatively poor with respect to the snow subtype of ground. In our implementation, we have assumed that the ground is smooth and the snow ground is white. However, the snow ground in natural images may not be smooth, and may vary in color. In addition, many other objects in natural images appear to be white and smooth. These factors negatively impact upon the performance with respect to snow ground.

\subsection{Experiments on combinations of scenery features}

Experiments were conducted to demonstrate the effectiveness of SceneryAnalyzer with combinations of scenery features.
The experiments involved all the images in the COREL and PhotoDisc databases. The top 20 images for each combination of scenery features were retrieved, and the precision and recall calculated; results are given in Table 2 . In this table, for each combination of scenery features, $\mathbf{F}$ denotes the set of images with the stipulated features, and $\mathbf{R}$ denotes the set of images retrieved. For a set $X,|X|$ denotes the number of elements in $X$. As a comparison, the performance of the five traditional CBIR techniques is given in Table 3 , where the keyblock model is abbreviated as $\mathrm{kb}$, the color histogram technique as ch, the color coherent vector as ccv, the Daubechies texture technique as daub, and the Haar texture technique as haar.

Table 2 demonstrates that the SceneryAnalyzer system is effective for the feature combinations listed. In contrast, Table 3 shows that traditional CBIR techniques are not satisfactory when handling combinations of semantic features. SceneryAnalyzer thus provides a new, semantics-based approach to handle queries for images.

Figures 21 and 22 provide sample images and their copies as annotated by SceneryAnalyzer.

\section{Conclusion and discussion}

In this paper, we have introduced the concept of the monotonic tree as a means to model high-level scenery features. Based on the monotonic tree representation, primitive elements of lowlevel features such as color, shape, and spatial location can be easily identified, clustered, and combined to form semantically meaningful regions (or features) for images. Thus, images can be automatically annotated with category keywords, including sky, building, tree, wave, lawn, water, snow, and ground. With this annotation, high-level (semantics-based) querying and browsing of images can be supported.

We made simple assumptions about the color, location, harshness, and shape of scenery features. Despite these simple assumptions, the proposed method achieved better results (as measured by precision-recall) than traditional CBIR techniques. These assumptions embody the domain knowledge about scenery images. If we combine the monotonic tree model with more complex domain knowledge, the accuracy of the system in extracting semantic features can be increased.

The reduced monotonic tree captures only the topological structure of the monotonic lines in an image. We may further define a differential slope and differential monotonic tree to capture differential information. A differential slope may be defined as a sequence of monotonic lines where the gradient is smooth. The differential monotonic tree constructed from these slopes will be capable of incorporating general domain knowledge. Thus, we may use the differential monotonic tree for general semantics extraction.

In future work, we intend to extend the proposed approach to categories other than scenery images. It may be cumbersome to provide a hand-crafted strategy for the identification of structural elements for each semantic feature. It will therefore be necessary for us to use machine-learning techniques to assist in the process of identifying structural elements for general semantic features. 
Table 2. Performance of SceneryAnalyzer on combinations of features

\begin{tabular}{lccccccccc}
\hline Features & $\begin{array}{c}\text { Sky } \\
\text { Tree }\end{array}$ & $\begin{array}{c}\text { Sky } \\
\text { Wave }\end{array}$ & $\begin{array}{c}\text { Tree } \\
\text { Building }\end{array}$ & $\begin{array}{c}\text { Tree } \\
\text { Ground }\end{array}$ & $\begin{array}{c}\text { Sky } \\
\text { Building } \\
\text { Lawn }\end{array}$ & $\begin{array}{c}\text { Sky } \\
\text { Tree } \\
\text { Ground }\end{array}$ & $\begin{array}{c}\text { Sky } \\
\text { Tree } \\
\text { Building } \\
\text { Lawn }\end{array}$ & $\begin{array}{c}\text { Tree } \\
\text { Water }\end{array}$ & $\begin{array}{c}\text { Sky } \\
\text { Snow }\end{array}$ \\
\hline$|\mathbf{F}|$ & 987 & 92 & 571 & 458 & 100 & 262 & 43 & 363 & 78 \\
$|\mathbf{R}|$ & 20 & 20 & 20 & 20 & 20 & 20 & 5 & 20 & 20 \\
$|\mathbf{F} \cap \mathbf{R}|$ & 17 & 17 & 18 & 18 & 18 & 18 & 5 & 11 & 8 \\
Precision & $85 \%$ & $85 \%$ & $90 \%$ & $90 \%$ & $90 \%$ & $90 \%$ & $100 \%$ & $55 \%$ & $40 \%$ \\
Recall & $1.7 \%$ & $18 \%$ & $3.1 \%$ & $3.9 \%$ & $18 \%$ & $6.9 \%$ & $12 \%$ & $3 \%$ & $10 \%$ \\
\hline
\end{tabular}

Table 3. Performance of the five traditional CBIR techniques on combinations of features

\begin{tabular}{|c|c|c|c|c|c|c|c|c|c|}
\hline Features & $\begin{array}{l}\text { Sky } \\
\text { Tree }\end{array}$ & $\begin{array}{c}\text { Sky } \\
\text { Wave }\end{array}$ & $\begin{array}{c}\text { Tree } \\
\text { Building }\end{array}$ & $\begin{array}{c}\text { Tree } \\
\text { Ground }\end{array}$ & $\begin{array}{c}\text { Sky } \\
\text { Building } \\
\text { Lawn }\end{array}$ & $\begin{array}{c}\text { Sky } \\
\text { Tree } \\
\text { Ground }\end{array}$ & $\begin{array}{c}\text { Sky } \\
\text { Tree } \\
\text { Building } \\
\text { Lawn }\end{array}$ & $\begin{array}{c}\text { Tree } \\
\text { Water }\end{array}$ & $\begin{array}{c}\text { Sky } \\
\text { Snow }\end{array}$ \\
\hline$|\overline{\mathbf{F}}|$ & 987 & 92 & 571 & 458 & 100 & 262 & 43 & 363 & 78 \\
\hline$\left|\mathbf{R}_{k b}\right|$ & 20 & 20 & 20 & 20 & 20 & 20 & 20 & 20 & 20 \\
\hline$\left|\mathbf{F} \cap \mathbf{R}_{k b}\right|$ & 7 & 5 & 8 & 3 & 5 & 3 & 0 & 3 & 1 \\
\hline Precision & $35 \%$ & $25 \%$ & $40 \%$ & $15 \%$ & $25 \%$ & $15 \%$ & $0 \%$ & $15 \%$ & $5 \%$ \\
\hline Recall & $0.7 \%$ & $5.4 \%$ & $1.4 \%$ & $0.7 \%$ & $5 \%$ & $1.1 \%$ & $0 \%$ & $0.8 \%$ & $1.3 \%$ \\
\hline$\left|\mathbf{R}_{c h}\right|$ & 20 & 20 & 20 & 20 & 20 & 20 & 20 & 20 & 20 \\
\hline$\left|\mathbf{F} \bigcap \mathbf{R}_{c h}\right|$ & 10 & 2 & 4 & 1 & 3 & 2 & 1 & 5 & 5 \\
\hline Precision & $50 \%$ & $10 \%$ & $20 \%$ & $5 \%$ & $15 \%$ & $10 \%$ & $5 \%$ & $25 \%$ & $25 \%$ \\
\hline Recall & $1 \%$ & $2.2 \%$ & $0.7 \%$ & $0.2 \%$ & $3 \%$ & $0.8 \%$ & $2.3 \%$ & $1.4 \%$ & $6.4 \%$ \\
\hline$\left|\mathbf{R}_{c c v}\right|$ & 20 & 20 & 20 & 20 & 20 & 20 & 20 & 20 & 20 \\
\hline$\left|\mathbf{F} \cap \mathbf{R}_{c c v}\right|$ & 10 & 1 & 8 & 5 & 0 & 2 & 0 & 7 & 0 \\
\hline Precision & $50 \%$ & $5 \%$ & $40 \%$ & $25 \%$ & $0 \%$ & $10 \%$ & $0 \%$ & $35 \%$ & $0 \%$ \\
\hline Recall & $1 \%$ & $1.1 \%$ & $1.4 \%$ & $1.1 \%$ & $0 \%$ & $0.8 \%$ & $0 \%$ & $1.9 \%$ & $0 \%$ \\
\hline$\left|\mathbf{R}_{\text {daub }}\right|$ & 20 & 20 & 20 & 20 & 20 & 20 & 20 & 20 & 20 \\
\hline$\left|\mathbf{F} \cap \mathbf{R}_{d a u b}\right|$ & 7 & 1 & 1 & 2 & 3 & 1 & 1 & 3 & 1 \\
\hline Precision & $35 \%$ & $5 \%$ & $5 \%$ & $10 \%$ & $15 \%$ & $5 \%$ & $5 \%$ & $15 \%$ & $5 \%$ \\
\hline Recall & $0.7 \%$ & $1.1 \%$ & $0.2 \%$ & $0.4 \%$ & $3 \%$ & $0.4 \%$ & $2.3 \%$ & $0.8 \%$ & $1.3 \%$ \\
\hline$\left|\mathbf{R}_{\text {haar }}\right|$ & 20 & 20 & 20 & 20 & 20 & 20 & 20 & 20 & 20 \\
\hline$\left|\mathbf{F} \cap \mathbf{R}_{\text {haar }}\right|$ & 7 & 3 & 5 & 2 & 1 & 2 & 1 & 7 & 1 \\
\hline Precision & $35 \%$ & $15 \%$ & $25 \%$ & $10 \%$ & $5 \%$ & $10 \%$ & $5 \%$ & $35 \%$ & $5 \%$ \\
\hline Recall & $0.7 \%$ & $3.3 \%$ & $0.9 \%$ & $0.4 \%$ & $1 \%$ & $0.8 \%$ & $2.3 \%$ & $1.9 \%$ & $1.3 \%$ \\
\hline
\end{tabular}

\section{References}

1. Ahuja N (1982) Dot pattern processing using voronoi neighborhoods. IEEE Trans Pattern Anal Mach Intell 4(3):336-343

2. Ahuja N, Rosenfeld A (1981) Mosaic models for texture. IEEE Trans Pattern Anal Mach Intell 3(1):1-11

3. Ahuja N, Tuceryan M (1989) Extraction of early perceptual structure in dot patterns: integrating region, boundary, and component gestalt. Comput Vision Graph Image Process 48(3):304356

4. Alshuth P, Hermes T, Klauck C, Krey J, Roper M (1996) Iris - image retrieval for images and videos. In: Proceedings First International Workshop of Image Databases and MultiMedia Search, Amsterdam, The Netherlands, pp 170-178

5. Bach JR, Fuller C, Gupta A, Hampapur A, Horowitz B, Jain R, Shu CF (1996) The virage image search engine: an open framework for image management. In: Proceedings SPIE, Storage and Retrieval for Still Image and Video Databases IV, San Jose, CA, pp 76-87

6. Crowley J, Parker A (1984) A representation of shape based on peaks and ridges in the difference of low-pass transform. IEEE Trans Pattern Anal Mach Intell 6(2):156-169

7. Dougherty ER, Pelz JB (1989) Texture-based segmentation by morphological granulometrics. In: Advanced Printing of Paper
Summaries, Electronic Imaging '89, vol 1, Boston, MA, pp 408414

8. Eakins JP (1996) Automatic image content retrieval - are we getting anywhere. In: Proceedings Third International Conference on Electronic Library and Visual Information Research, pp $123-135$

9. Faloutsos C, Barber R, Flickner M, Hafner J, Niblack W, Petkovic D, Equitz W (1994) Efficient and effective querying by image content. J Intell Inf Syst 3(3/4):231-262

10. Flickner M, Sawhney H, Niblack W, Ashley J, Huang Q, Dom B, et al (1995) Query by image and video content: the QBIC system. IEEE Comput 28(9):23-32

11. Forsyth DA, Malik J, Fleck MM, Greenspan H, Leung T, Belongie S, Carson C, Bregler C (1996) Finding pictures of objects in large collections of images. In: Report of the NSF/ARPA Workshop on 3D Object Representation for Computer Vision, p 335

12. Hirata K, Kato T (1993) Rough sketch-based image information retrieval. NEC Res Dev 34(2):263-273

13. Klaus B, Horn P (1988) Robot Vision, 4th edn. MIT Press, Cambridge, MA

14. Fries RW, Modestino JW, Vickers AL (1981) Texture discrimination based upon an assumed stochastic texture model. IEEE Trans Pattern Anal Mach Intell 3(5):557-580 
15. Korn F, Sidiropoulos N, Faloutsos C, Siegel E, Protopapas Z (1996) Fast nearest-neighbor search in medical image databases. In: Conference on Very Large Data Bases (VLDB96)

16. Zhu L, Rao A, Zhang A (2000) Theory of keyblock-based image retrieval. ACM Transactions on Information Systems, Vol.20, No. 2, pp 224-258, April

17. Lindeberg T (1994) Scale-space theory in computer vision. Kluwer, Dordrecht

18. Ma WY, Manjunath BS (1997) Netra: A toolbox for navigating large image databases. In: International Conference on Image Processing pages, I:568-571

19. Mallat S (1989) A theory for multiresolution signal decomposition: the wavelet representation. IEEE Trans Pattern Anal Mach Intell 11:674-693

20. Mandelbrot BB (1977) Fractals - form, chance, dimension. W.H. Freeman, San Francisco, CA

21. Manjunath BS, Ma WY (1996) Texture features for browsing and retrieval of image data. IEEE Trans Pattern Anal Mach Intell 18(8):837-842

22. Mehrotra R, Gary JE (1995) Similar-shape retrieval in shape data management. IEEE Comput 28(9):57-62

23. Mokhtarian F, Abbasi S, Kittler J (1996) Efficient and robust retrieval by shape content through curvature scale space. In: Proceedings International Workshop on Image Databases and MultiMedia Search, Amsterdam, The Netherlands, pp 35-42

24. Mokhtarian F, Abbasi S, Kittler J (1996) Robust and efficient shape indexing through curvature scale space. In: Proceedings British Machine Vision Conference, Edinburgh, UK, pp 53-62

25. Morse SP (1969) Concepts of use in computer map processing. Commun ACM 12(3):147-152

26. Pass G, Zabih R, Miller J (1996) Comparing images using color coherence vectors. In: Proceedings ACM Multimedia '96, Boston, MA, pp 65-73

27. Pentland A, Picard R, Sclaroff S (1994) Photobook: tools for content-based manipulation of image databases. In: Proceedings SPIE Conference on Storage and Retrieval of Image and Video Databases II, pp 34-47

28. Picard R (1996) A society of models for video and image libraries. Technical Report 360, MIT Media Laboratory Perceptual Computing

29. Robl C, Farber G (1998) Contour tracer for a fast and precise edge-line extraction. In: IAPR Workshop On Machine Vision Applications (MVA98)

30. Rosin PL, West (1989) Segmentation of edges into lines and arcs. Image Vision Comput 7(2):109-114

31. Rosin PL, West GAW (1992) Multi-stage combined ellipse and line detection. In: British Machine Vision Conference (BMVC92), pp 197-206

32. Roubal J, Poiker TK (1985) Automated contour labelling and the contour tree. In: Proceedings AUTO-CARTO 7 pp 472-481

33. Safar M, Shahabi C, Sun X (2000) Image retrieval by shape: a comparative study. In: Proceedings IEEE International Conference on Multimedia and Exposition (ICME), USA
34. Shahabi C, Safar M (1999) Efficient retrieval and spatial querying of 2D objects. In: IEEE International Conference on Multimedia Computing and Systems (ICMCS99), Florence, Italy, pp 611-617

35. Sheikholeslami G, Zhang A (1997) An approach to clustering large visual databases using wavelet transform. In: Proceedings SPIE Conference on Visual Data Exploration and Analysis IV, San Jose, CA, pp 322-333

36. Smith JR, Chang S (1994) Transform features for texture classification and discrimination in large image databases. In: Proceedings IEEE International Conference on Image Processing, pp 407-411

37. Smith JR, Chang SF (1996) VisualSeek: a fully automated content-based image query system. In: Proceedings ACM Multimedia '96, Boston, MA, pp 87-98

38. Snyder WE, Qi H, Sander W (1999) A hexagonal coordinate system. SPIE Med Imaging: Image Process 1-2:716-727

39. Song Y, Zhang A (2002) Monotonic tree. In: 10th International Conference on Discrete Geometry for Computer Imagery, Bordeaux, France

40. Strang G, Nguyen T (1996) Wavelets and filter banks. WellesleyCambridge Press, Wellesley, MA

41. Stromberg WD, Farr TG (1986) A Fourier-based textural feature extraction procedure. IEEE Trans Geosci Remote Sensing 24(5):722-732

42. Swain MJ, Ballard D (1991) Color indexing. Int J Comput Vision 7(1):11-37

43. Syeda-Mahmood TF (1996) Finding shape similarity using a constrained non-rigid transform. In: International Conference on Pattern Recognition

44. Tao Y, Grosky WI (1999) Delaunay triangulation for image object indexing: a novel method for shape representation. In: Proceedings Seventh SPIE Symposium on Storage and Retrieval for Image and Video Databases, San Jose, CA, pp 631-942

45. Torralba AB, Oliva A (1999) Semantic organization of scenes using discriminant structural templates. In: International Conference on Computer Vision (ICCV99), pp 1253-1258

46. van Kreveld M, van Oostrum R, Bajaj C, Pascucci V, Schikore D (1997) Contour trees and small seed sets for iso-surface traversal. In: Proceedings 13th ACM Symposium on Computational Geometry, pp 212-220

47. Wang J, Acharya R (1998) Efficient access to and retrieval from a shape image database. In: IEEE Workshop on Content Based Access of Image and Video Libraries (CBAIL 98), Santa Barbara, CA

48. Wang J, Acharya R (1998) A vertex based shape coding approach for similar shape retrieval. In: ACM Symposium on Applied Computing, Atlanta, GA, pp 520-524

49. Zahn CT (1971) Graph-theoretical methods for detecting and describing gestalt clusters. IEEE Trans Comput C-20:68-86 